\title{
A Low-Complexity Time-Domain MMSE Channel Estimator for Space-Time/Frequency Block-Coded OFDM Systems
}

\author{
Habib Şenol, ${ }^{1}$ Hakan Ali Çırpan, ${ }^{2}$ Erdal Panayırcı, ${ }^{3}$ and Mesut Çevik ${ }^{2}$ \\ ${ }^{1}$ Department of Computer Engineering, Kadir Has University, Cibali 34230, Istanbul, Turkey \\ ${ }^{2}$ Department of Electrical Engineering, Istanbul University, Avcilar 34850, Istanbul, Turkey \\ ${ }^{3}$ Department of Electrical and Electronics Engineering, Bilkent University, Bilkent 06800, Ankara, Turkey
}

Received 1 June 2005; Revised 8 February 2006; Accepted 18 February 2006

\begin{abstract}
Focusing on transmit diversity orthogonal frequency-division multiplexing (OFDM) transmission through frequency-selective channels, this paper pursues a channel estimation approach in time domain for both space-frequency OFDM (SF-OFDM) and space-time OFDM (ST-OFDM) systems based on AR channel modelling. The paper proposes a computationally efficient, pilotaided linear minimum mean-square-error (MMSE) time-domain channel estimation algorithm for OFDM systems with transmitter diversity in unknown wireless fading channels. The proposed approach employs a convenient representation of the channel impulse responses based on the Karhunen-Loeve (KL) orthogonal expansion and finds MMSE estimates of the uncorrelated KL series expansion coefficients. Based on such an expansion, no matrix inversion is required in the proposed MMSE estimator. Subsequently, optimal rank reduction is applied to obtain significant taps resulting in a smaller computational load on the proposed estimation algorithm. The performance of the proposed approach is studied through the analytical results and computer simulations. In order to explore the performance, the closed-form expression for the average symbol error rate (SER) probability is derived for the maximum ratio receive combiner (MRRC). We then consider the stochastic Cramer-Rao lower bound(CRLB) and derive the closed-form expression for the random KL coefficients, and consequently exploit the performance of the MMSE channel estimator based on the evaluation of minimum Bayesian MSE. We also analyze the effect of a modelling mismatch on the estimator performance. Simulation results confirm our theoretical analysis and illustrate that the proposed algorithms are capable of tracking fast fading and improving overall performance.
\end{abstract}

Copyright $\odot 2006$ Hindawi Publishing Corporation. All rights reserved.

\section{INTRODUCTION}

Next generations of broadband wireless communications systems aim to support different types of applications with a high quality of service and high-data rates by employing a variety of techniques capable of achieving the highest possible spectrum efficiency [1]. The fulfilment of the constantly increasing demand for high-data rate and high quality of service requires the use of much more spectrally efficient and flexible modulation and coding techniques, with greater immunity against severe frequency-selective fading. The combined application of OFDM and transmit antenna diversity appears to be capable of enabling the types of capacities and data rates needed for broadband wireless services [2-8].

OFDM has emerged as an attractive and powerful alternative to conventional modulation schemes in the recent past due to its various advantages in lessening the severe effect of frequency-selective fading. The broadband channel undergoes severe multipath fading, the equalizer in a conventional single-carrier modulation becomes prohibitively complex to implement. OFDM is therefore chosen over a single-carrier solution due to lower complexity of equalizers [1]. In OFDM, the entire signal bandwidth is divided into a number of narrowbands or orthogonal subcarriers, and signal is transmitted in the narrowbands in parallel. Therefore, it reduces intersymbol interference (ISI), obviates the need for complex equalization, and thus greatly simplifies channel estimation/equalization task. Moreover, its structure also allows efficient hardware implementations using fast Fourier transform (FFT) and polyphase filtering [2]. On the other hand, due to dispersive property of the wireless channel, subcarriers on those deep fades may be severely attenuated. To robustify the performance against deep fades, diversity techniques have to be used. Transmit antenna diversity is an effective technique for combatting fading in mobile in multipath wireless channels $[4,9]$. Among a number of antenna diversity methods, the Alamouti method is very simple to implement [9]. This is an example for space-time block code (STBC) for two transmit antennas, and the simplicity of the receiver is attributed to the orthogonal nature of the code 
$[10,11]$. The orthogonal structure of these space-time block codes enable the maximum likelihood decoding to be implemented in a simple way through decoupling of the signal transmitted from different antennas rather than joint detection resulting in linear processing [9].

The use of OFDM in transmitter diversity systems motives exploitation of diversity dimensions. Inspired by this fact, a number of coding schemes have been proposed recently to achieve maximum diversity gain [6-8]. Among them, ST-OFDM has been proposed recently for delay spread channels. On the other hand, transmitter OFDM also offers the possibility of coding in a form of SF-OFDM [6-8]. OFDM maps the frequency-selective channel into a set of flat fading subchannels, whereas space-time/frequency encoding/decoding facilitates equalization and achieves performance gains by exploiting the diversity available with transmit antennas. Moreover, SF-OFDM and ST-OFDM transmitter diversity systems were compared in [6], under the assumption that the channel responses are known or can be estimated accurately at the receiver. It was shown that the SFOFDM system has the same performance as a previously reported ST-OFDM scheme in slow fading environments but shows better performance in the more difficult fast fading environments. Also, since, SF-OFDM transmitter diversity scheme performs the decoding within one OFDM block, it only requires half of the decoder memory needed for the STOFDM system of the same block size. Similarly, the decoder latency for SF-OFDM is also half that of the ST-OFDM implementation.

Channel estimation for transmit diversity OFDM systems has attracted much attention with pioneering works by $\mathrm{Li}$ et al. [4] and $\mathrm{Li}$ [5]. A robust channel estimator for OFDM systems with transmitter diversity has been first developed with the temporal estimation by using the correlation of the channel parameters at different frequencies [4]. Its simplified approaches have been then presented by identifying significant taps [5]. Among many other techniques, pilot-aided MMSE estimation was also applied in the context of space-time block coding (STBC) either in the time domain for the estimation of channel impulse response (CIR) $[12,13]$ or in the frequency domain for the estimation of transfer function (TF) [14]. However channel estimation in the time domain turns out to be more efficient since the number of unknown parameters is greatly decreased compared to that in the frequency domain. Focusing on transmit diversity OFDM transmissions through frequency-selective fading channels, this paper pursues a time-domain MMSE channel estimation approach for both SF-OFDM and STOFDM systems. We derive a low complexity MMSE channel estimation algorithm for both transmiter diversity OFDM systems based on AR channel modelling. In the development of the MMSE channel estimation algorithm, the channel taps are assumed to be random processes. Moreover, orthogonal series representation based on the KL expansion of a random process is applied which makes the expansion coefficient random variables uncorrelated $[15,16]$. Thus, the algorithm estimates the uncorrelated complex expansion coefficients using the MMSE criterion.
The layout of the paper is as follows. In Section 2, a general model for transmit diversity OFDM systems together with SF and ST coding, AR channel modelling, and unified signal model are presented. In Section 3, an MMSE channel estimation algorithm is developed for the KL expansion coefficients. Performance of the proposed algorithm is studied based on the evaluation of the modified Cramer-Rao bound of the channel parameters and the SNR and correlation mismatch analysis together with closed-form expression for the average SER probability in Section 4 . Some simulation examples are provided in Section 5. Finally, conclusions are drawn in Section 6.

\section{SYSTEM MODEL}

\subsection{Alamouti's transmit diversity scheme for OFDM systems}

In this paper, we consider a transmitter diversity scheme in conjunction with OFDM signaling. Many transmit diversity schemes have been proposed in the literature offering different complexity versus performance trade-offs. We choose Alamouti's transmit diversity scheme due to its simple implementation and good performance [9]. The Alamouti's scheme imposes an orthogonal spatio-temporal structure on the transmitted symbols that guarantees full (i.e., order 2) spatial diversity.

We consider the Alamouti transmitter diversity coding scheme, employed in an OFDM system utilizing $K$ subcarriers per antenna transmissions. Note that $K$ is chosen as an even integer. The fading channel between the $\mu$ th transmit antenna and the receive antenna is assumed to be frequency selective and is described by the discrete-time baseband equivalent impulse response $\mathbf{h}_{\mu}(n)=\left[h_{\mu, 0}(n), \ldots, h_{\mu, L}(n)\right]^{T}$, with $L$ standing for the channel order.

At each time index $n$, the input serial information symbols with symbol duration $T_{s}$ are converted into a data vector $\mathbf{X}(n)=[X(n, 0), \ldots, X(n, K-1)]^{T}$ by means of a serialto-parallel converter. Its block duration is $K T_{s}$. Moreover, $X(n, k)$ denote the $k$ th forward polyphase component of the serial data symbols, that is, $X(n, k)=X(n K+k)$ for $k=$ $0,1,2, \ldots, K-1$ and $n=0,1,2, \ldots, N-1$. Polyphase component $X(n, k)$ can also be viewed as the data symbol to be transmitted on the $k$ th tone during the block instant $n$. The transmitter diversity encoder arranges $\mathbf{X}(n)$ into two vectors $\mathbf{X}_{1}(n)$ and $\mathbf{X}_{2}(n)$ according to an appropriate coding scheme described in $[6,9]$. The coded vector $\mathbf{X}_{1}(n)$ is modulated by an IFFT into an OFDM sequence. Then cyclic prefix is added to the OFDM symbol sequence, and the resulting signal is transmitted through the first transmit antenna. Similarly, $\mathbf{X}_{2}(n)$ is modulated by IFFT, cyclically extended, and transmitted from the second transmit antenna.

At the receiver side, the antenna receives a noisy superposition of the transmissions through the fading channels. We assume ideal carrier synchronization, timing, and perfect symbol-rate sampling, and the cyclic prefix is removed at the receiver end. 


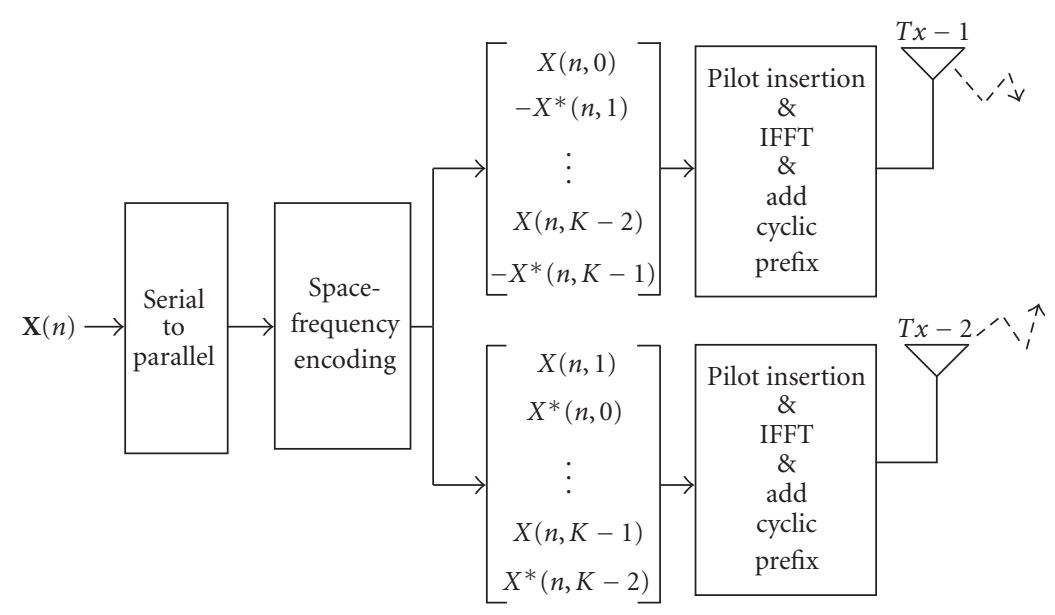

FIGURE 1: Space-frequency coding on two adjacent FFT frequency bins.

The generation of coded vectors $\mathbf{X}_{1}(n)$ and $\mathbf{X}_{2}(n)$ from the information symbols leads to corresponding transmit diversity OFDM scheme. In our system, the generation of $\mathbf{X}_{1}(n)$ and $\mathbf{X}_{2}(n)$ is performed via the space-frequency coding and space-time coding, respectively, which were first suggested in [9] and later generalized in [7, 8].

\section{Space-frequency coding}

We first consider a strategy which basically consists of coding across OFDM tones and is therefore called space-frequency coding [6-8]. Resorting to coding across tones, the set of generally correlated OFDM subchannels is first divided into groups of subchannels. This subchannel grouping with appropriate system parameters does preserve diversity gain while simplifying not only the code construction but decoding algorithm significantly as well [6]. A block diagram of a two-branch space-frequency OFDM transmitter diversity system is shown in Figure 1. Resorting subchannel grouping, $\mathbf{X}(n)$ is coded into two vectors $\mathbf{X}_{1}(n)$ and $\mathbf{X}_{2}(n)$ by the spacefrequency encoder as

$$
\begin{aligned}
\mathbf{X}_{1}(n)= & {\left[X(n, 0),-X^{*}(n, 1), \ldots, X(n, K-2),\right.} \\
& \left.-X^{*}(n, K-1)\right]^{T} \\
\mathbf{X}_{2}(n)= & {\left[X(n, 1), X^{*}(n, 0), \ldots, X(n, K-1),\right.} \\
& \left.X^{*}(n, K-2)\right]^{T}
\end{aligned}
$$

where $(\cdot)^{*}$ stands for complex conjugation. In spacefrequency Alamouti scheme, $\mathbf{X}_{1}(n)$ and $\mathbf{X}_{2}(n)$ are transmitted through the first and second antenna elements, respectively, during the OFDM block instant $n$.

The operations of the space-frequency block encoder can best be described in terms of even and odd polyphase component vectors. If we denote even and odd component vectors of $\mathbf{X}(n)$ as

$$
\begin{aligned}
& \mathbf{X}_{e}(n)=[X(n, 0), X(n, 2), \ldots, X(n, K-4), X(n, K-2)]^{T}, \\
& \mathbf{X}_{o}(n)=[X(n, 1), X(n, 3), \ldots, X(n, K-3), X(n, K-1)]^{T},
\end{aligned}
$$

then the space-frequency block code transmission matrix may be represented by

$$
\begin{gathered}
\text { Space } \longrightarrow \\
\text { Frequency } \downarrow\left[\begin{array}{cc}
\mathbf{X}_{e}(n) & \mathbf{X}_{o}(n) \\
-\mathbf{X}_{o}^{*}(n) & \mathbf{X}_{e}^{*}(n)
\end{array}\right] .
\end{gathered}
$$

If the received signal sequence is parsed in even and odd blocks of $K / 2$ tones, $\mathbf{Y}_{e}(n)=[Y(n, 0), Y(n, 2), \ldots, Y(n, K-$ 2) $]^{T}$ and $\mathbf{Y}_{o}(n)=[Y(n, 1), Y(n, 3), \ldots, Y(n, K-1)]^{T}$, the received signal can be expressed in vector form as

$$
\begin{gathered}
\mathbf{Y}_{e}(n)=\mathcal{X}_{e}(n) \mathbf{H}_{1, e}(n)+\mathcal{X}_{o}(n) \mathbf{H}_{2, e}(n)+\mathbf{W}_{e}(n), \\
\mathbf{Y}_{o}(n)=-\mathcal{X}_{o}^{\dagger}(n) \mathbf{H}_{1, o}(n)+\mathcal{X}_{e}^{\dagger}(n) \mathbf{H}_{2, o}(n)+\mathbf{W}_{o}(n),
\end{gathered}
$$

where $\mathcal{X}_{e}(n)$ and $\mathcal{X}_{o}(n)$ are $K / 2 \times K / 2$ diagonal matrices whose elements are $\mathbf{X}_{e}(n)$ and $\mathbf{X}_{o}(n)$, respectively, and $(\cdot)^{\dagger}$ denotes conjugate transpose. Let $\mathbf{H}_{\mu, e}(n)=\left[H_{\mu}(n, 0)\right.$, $\left.H_{\mu}(n, 2), \ldots, H_{\mu}(n, K-2)\right]^{T}$ and $\mathbf{H}_{\mu, o}(n)=\left[H_{\mu}(n, 1)\right.$, $\left.H_{\mu}(n, 3), \ldots, H_{\mu}(n, K-1)\right]^{T}$ be $K / 2$ length vectors denoting the even and odd component vectors of the channel attenuations between the $\mu$ th transmitter and the receiver. Finally, $\mathbf{W}_{e}(n)$ and $\mathbf{W}_{o}(n)$ are zero-mean, i.i.d. Gaussian vectors with covariance matrix $\sigma^{2} \mathbf{I}_{K / 2}$.

\section{Space-time coding}

In contrast to SF-OFDM coding, ST encoder maps every two consecutive symbol blocks $\mathbf{X}(n)$ and $\mathbf{X}(n+1)$ to the following $2 K \times 2$ matrix:

$$
\begin{gathered}
\text { Space } \longrightarrow \\
\text { Time } \downarrow\left[\begin{array}{cc}
\mathbf{X}(n) & \mathbf{X}(n+1) \\
-\mathbf{X}^{*}(n+1) & \mathbf{X}^{*}(n)
\end{array}\right] .
\end{gathered}
$$




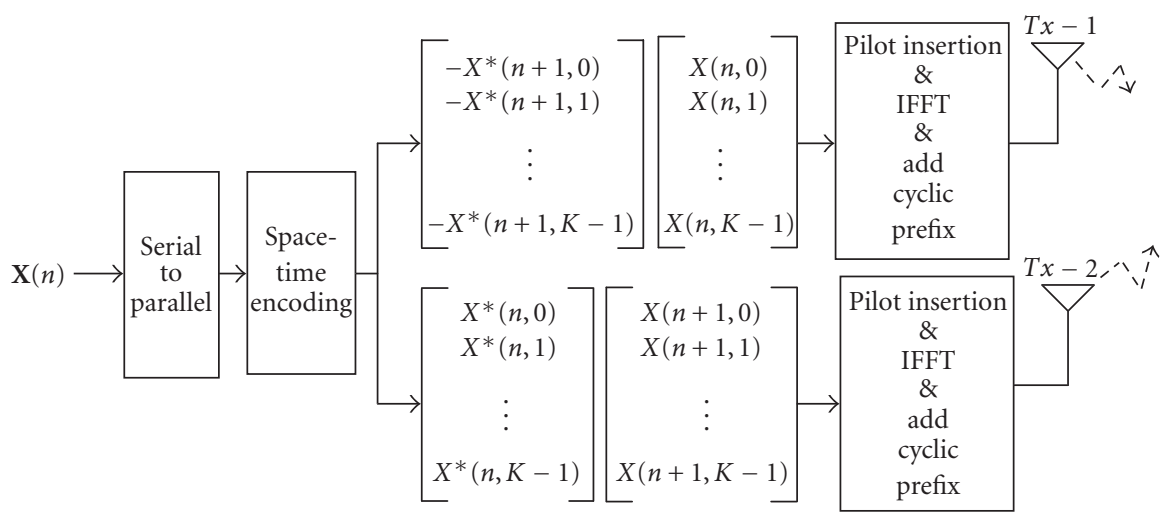

FIGURE 2: Space-time coding on two adjacent OFDM blocks.

The columns are transmitted in successive time intervals with the upper and lower blocks in a given column sent simultaneously through the first and second transmit antennas, respectively, as shown in Figure 2. If we focus on each received block separately, each pair of two consecutive received blocks $\mathbf{Y}(n)=[Y(n, 0), \ldots, Y(n, K-1)]^{T}$ and $\mathbf{Y}(n+1)=$ $[Y(n+1,0), \ldots, Y(n+1, K-1)]^{T}$ are given by

$$
\begin{aligned}
\mathbf{Y}(n)= & \mathcal{X}(n) \mathbf{H}_{1}(n) \\
& +\mathcal{X}(n+1) \mathbf{H}_{2}(n)+\mathbf{W}(n), \\
\mathbf{Y}(n+1)= & -\mathcal{X}^{\dagger}(n+1) \mathbf{H}_{1}(n+1) \\
& +\mathcal{X}^{\dagger}(n) \mathbf{H}_{2}(n+1)+\mathbf{W}(n+1),
\end{aligned}
$$

where $\mathcal{X}(n)$ and $\mathcal{X}(n+1)$ are $K \times K$ diagonal matrices whose elements are $\mathbf{X}(n)$ and $\mathbf{X}(n+1)$, respectively. $\mathbf{H}_{\mu}(n)$ is the channel frequency response between the $\mu$ th transmitter and the receiver antenna at the $n$th time slot which is obtained from channel impulse response $\mathbf{h}_{\mu}(n)$. Finally, $\mathbf{W}(n)$ and $\mathbf{W}(n+1)$ are zero-mean, i.i.d. Gaussian vectors with covariance matrix $\sigma^{2} \mathbf{I}_{K}$ per dimension.

Having specified the received signal models (4) and (6), we proceed to explore channel models.

\subsection{AR models considerations}

Channel estimation in transmit diversity systems results in ill-posed problem since for every incoming signal, extra unknowns appear. However, imposing structure on channel variations render estimation problem tractable. Fortunately many wireless channels exhibit structured variations hence fit into some evolution model. Among different models, the AR model is adopted herein for channel dynamics. Since only the first few correlation terms are important to finitely parametrize structured variations of a wireless channel in the design of a channel estimator, low-order AR models can capture most of the channel tap dynamics and lead to effective estimation techniques. Thus this paper associates channel effect in SF/ST-OFDM systems with a first-order AR process.

\section{AR channel model in SF-OFDM}

The even and odd component vectors of the channels $\mathbf{H}_{\mu, e}(n)$ and $\mathbf{H}_{\mu, o}(n)$ between the $\mu$ th transmitter and the receiver can be modelled as a first-order AR process. An AR process can be represented as

$$
\mathbf{H}_{\mu, o}(n)=\alpha \mathbf{H}_{\mu, e}(n)+\boldsymbol{\eta}_{\mu, o}(n),
$$

where $\alpha$ can be obtained from the normalized exponential discrete channel correlation for different subcarriers in SFOFDM case. Moreover, using (7), simple manipulations lead to the covariance matrix $\mathbf{C}_{\boldsymbol{\eta}_{\mu, o}}(n)=\left(1-|\alpha|^{2}\right) \mathbf{I}_{K / 2}$ of zeromean Gaussian AR process noise $\boldsymbol{\eta}_{\mu, o}(n)$.

\section{AR channel model in ST-OFDM}

Similarly, the channel frequency response $\mathbf{H}_{\mu}(n)$ between the $\mu$ th transmitter and the receiver antenna at the $n$th time slot varies accordingly:

$$
\mathbf{H}_{\mu}(n+1)=\alpha \mathbf{H}_{\mu}(n)+\boldsymbol{\eta}_{\mu}(n+1),
$$

where $\alpha$ is related to Doppler frequency $f_{d}$ and symbol duration $T_{s}$ via $\alpha=J_{o}\left(2 \pi f_{d} T_{s}\right)$ in ST-OFDM. Using (8), we obtain the covariance matrix of zero-mean Gaussian AR process noise $\boldsymbol{\eta}_{\mu}(n+1)$ as $\mathbf{C}_{\boldsymbol{\eta}_{\mu}(n+1)}=\left(1-|\alpha|^{2}\right) \mathbf{I}_{K}$.

\subsection{Unifying SF-OFDM and ST-OFDM signal models}

The transmitter diversity OFDM schemes considered here can be unified into one general model for channel estimation. Considering signal models (4) and (6) with corresponding AR models (7) and (8), we unify SF-OFDM and STOFDM in the following equivalent model:

$$
\left[\begin{array}{l}
\mathbf{Y}_{1} \\
\mathbf{Y}_{2}
\end{array}\right]=\left[\begin{array}{cc}
X_{1} & X_{2} \\
-\mathcal{X}_{2}^{\dagger} & X_{1}^{\dagger}
\end{array}\right]\left[\begin{array}{l}
\mathbf{H}_{1} \\
\mathbf{H}_{2}
\end{array}\right]+\left[\begin{array}{l}
\mathbf{W}_{1} \\
\mathbf{W}_{2}
\end{array}\right]
$$


For convenience, we list the corresponding vectors and matrices for SF-OFDM as

$$
\begin{aligned}
& {\left[\frac{\mathbf{Y}_{1}}{\mathbf{Y}_{2}}\right]=\left[\frac{\mathbf{Y}_{e}(n)}{\mathbf{Y}_{o}(n) / \alpha}\right],} \\
& {\left[\begin{array}{c|c}
X_{1} & X_{2} \\
\hline-X_{2}^{\dagger} & X_{1}^{\dagger}
\end{array}\right]=\left[\begin{array}{c|c}
X_{e}(n) & X_{o}(n) \\
\hline-X_{o}^{\dagger}(n) & X_{e}^{\dagger}(n)
\end{array}\right]} \\
& {\left[\frac{\mathbf{H}_{1}}{\mathbf{H}_{2}}\right]=\left[\frac{\mathbf{H}_{1, e}(n)}{\mathbf{H}_{2, e}(n)}\right],} \\
& {\left[\frac{\mathbf{W}_{1}}{\mathbf{W}_{2}}\right]=\left[\frac{\mathbf{W}_{e}(n)}{1 / \alpha\left[\mathbf{W}_{o}(n)-\mathcal{X}_{o}^{\dagger}(n) \boldsymbol{\eta}_{1, o}(n)+\mathcal{X}_{e}^{\dagger}(n) \boldsymbol{\eta}_{2, o}(n)\right]}\right] \text {, }}
\end{aligned}
$$

where $\mathbf{W}_{1} \sim \mathcal{N}\left(\mathbf{0}, \sigma^{2} \mathbf{I}_{K / 2}\right), \mathbf{W}_{2} \sim \mathcal{N}\left(\mathbf{0}, \sigma^{2}+2\left(1-|\alpha|^{2}\right) /\right.$ $\left.|\alpha|^{2} \mathbf{I}_{K / 2}\right)$. Similarly for ST-OFDM,

$$
\begin{aligned}
& {\left[\frac{\mathbf{Y}_{1}}{\mathbf{Y}_{2}}\right]=\left[\frac{\mathbf{Y}(n)}{\mathbf{Y}(n+1) / \alpha}\right],} \\
& {\left[\begin{array}{c|c}
X_{1} & X_{2} \\
\hline-X_{2}^{\dagger} & X_{1}^{\dagger}
\end{array}\right]=\left[\begin{array}{c|c}
\mathcal{X}(n) & \mathcal{X}(n+1) \\
\hline-\mathcal{X}^{\dagger}(n+1) & \mathcal{X}^{\dagger}(n)
\end{array}\right],} \\
& {\left[\frac{\mathbf{H}_{1}}{\mathbf{H}_{2}}\right]=\left[\frac{\mathbf{H}_{1}(n)}{\mathbf{H}_{2}(n)}\right],} \\
& {\left[\frac{\mathbf{W}_{1}}{\mathbf{W}_{2}}\right]} \\
& =\left[\frac{\mathbf{W}(n)}{1 / \alpha\left[\mathbf{W}(n+1)-\mathcal{X}^{\dagger}(n+1) \boldsymbol{\eta}_{1}(n+1)+\mathcal{X}^{\dagger}(n) \boldsymbol{\eta}_{2}(n+1)\right]}\right] .
\end{aligned}
$$

Note that $\mathbf{W}_{1} \sim \mathcal{N}\left(\mathbf{0}, \sigma^{2} \mathbf{I}_{K}\right)$ and $\mathbf{W}_{2} \sim \mathcal{N}\left(\mathbf{0}, \sigma^{2}+2\left(1-|\alpha|^{2}\right) /\right.$ $\left.|\alpha|^{2} \mathbf{I}_{K}\right)$.

Relying on the unifying model (9), we will develop a channel estimation algorithm according to the MMSE criterion and then explore the performance of the estimator. An MMSE approach adapted herein explicitly models the channel parameters by the KL series representation since KL expansion allows one to tackle the estimation of correlated parameters as a parameter estimation problem of the uncorrelated coefficients.

\section{MMSE ESTIMATION}

Pilots-symbols-assisted techniques can provide information about an undersampled version of the channel that may be easier to identify. In this paper, we therefore address the problem of estimating channel parameters by exploiting the distributed training symbols.

\subsection{MMSE estimation of the multipath channels}

Since both SF and ST block-coded OFDM systems have symmetric structure in frequency and time, respectively, the pilot symbols should be uniformly placed in pairs. Specifically, we also assume that even number of symbols are placed between pilot pairs for SF-OFDM systems. Based on these pilot structures, (9) is modified to represent the signal model corresponding to pilot symbols as follows:

$$
\underbrace{\left[\begin{array}{l}
\mathbf{Y}_{1, p} \\
\mathbf{Y}_{2, p}
\end{array}\right]}_{\mathbf{Y}_{p}}=\underbrace{\left[\begin{array}{cc}
\boldsymbol{X}_{1, p} & \boldsymbol{X}_{2, p} \\
-\boldsymbol{X}_{2, p}^{\dagger} & \boldsymbol{X}_{1, p}^{\dagger}
\end{array}\right]}_{\overline{\boldsymbol{X}}_{p}} \underbrace{\left[\begin{array}{l}
\mathbf{H}_{1, p} \\
\mathbf{H}_{2, p}
\end{array}\right]}_{\mathbf{H}_{p}}+\underbrace{\left[\begin{array}{l}
\mathbf{W}_{1, p} \\
\mathbf{W}_{2, p}
\end{array}\right]}_{\mathbf{W}_{p}},
$$

where $(\cdot)_{p}$ is introduced to represent the vectors corresponding to pilot locations.

For a class of QPSK-modulated pilot symbols, the new observation model can be formed by premultiplying both sides of (12) by $\bar{X}_{p}^{\dagger}$ :

$$
\bar{x}_{p}^{\dagger} \mathbf{Y}_{p}=\bar{x}_{p}^{\dagger} \bar{x}_{p} \mathbf{H}_{p}+\bar{x}_{p}^{\dagger} \mathbf{w}_{p} .
$$

Since $\bar{X}_{p}^{\dagger} \bar{X}_{p}=2 \mathbf{I}_{2 K_{p}}$, and letting $\tilde{\mathbf{Y}}_{p}=\bar{X}_{p}^{\dagger} \mathbf{Y}_{p}$ and $\widetilde{\mathbf{W}}_{p}=$ $\overline{\mathcal{X}}_{p}^{\dagger} \mathbf{W}_{p}$, (13) can be rewritten as

$$
\tilde{\mathbf{Y}}_{p}=2 \mathbf{H}_{p}+\widetilde{\mathbf{W}}_{p}
$$

namely,

$$
\left[\begin{array}{c}
\widetilde{\mathbf{Y}}_{1, p} \\
\widetilde{\mathbf{Y}}_{2, p}
\end{array}\right]=2\left[\begin{array}{l}
\mathbf{H}_{1, p} \\
\mathbf{H}_{2, p}
\end{array}\right]+\left[\begin{array}{c}
\widetilde{\mathbf{W}}_{1, p} \\
\widetilde{\mathbf{W}}_{2, p}
\end{array}\right],
$$

where

$$
\begin{aligned}
\widetilde{\mathbf{Y}}_{1, p} & =\mathcal{X}_{1, p}^{\dagger} \mathbf{Y}_{1, p}-\mathcal{X}_{2, p} \mathbf{Y}_{2, p}, \\
\widetilde{\mathbf{Y}}_{2, p} & =\mathcal{X}_{2, p}^{\dagger} \mathbf{Y}_{1, p}+\mathcal{X}_{1, p} \mathbf{Y}_{2, p}, \\
\widetilde{\mathbf{W}}_{1, p} & =X_{1, p}^{\dagger} \mathbf{W}_{1, p}-\mathcal{X}_{2, p} \mathbf{W}_{2, p}, \\
\widetilde{\mathbf{W}}_{2, p} & =X_{2, p}^{\dagger} \mathbf{W}_{1, p}+X_{1, p} \mathbf{W}_{2, p},
\end{aligned}
$$

and note that $\widetilde{\mathbf{W}}_{1, p} \sim \mathcal{N}\left(\mathbf{0}, \bar{\sigma}^{2} \mathbf{I}_{K_{p}}\right)$ and $\widetilde{\mathbf{W}}_{2, p} \sim \mathcal{N}\left(\mathbf{0}, \bar{\sigma}^{2} \mathbf{I}_{K_{p}}\right)$ where $\bar{\sigma}^{2}=\left(\sigma^{2}\left(1+|\alpha|^{2}\right)+2\left(1-|\alpha|^{2}\right)\right) /|\alpha|^{2}$. By writing each row of (16) separately, we obtain the following observation equation set to estimate the channels $\mathbf{H}_{1, p}$ and $\mathbf{H}_{2, p}$ :

$$
\tilde{\mathbf{Y}}_{\mu, p}=2 \mathbf{H}_{\mu, p}+\widetilde{\mathbf{W}}_{\mu, p} \quad \mu=1,2 .
$$

Since our goal is to develop channel estimation in time domain, (17) can be expressed in terms of $\mathbf{h}_{\mu}$ by using $\mathbf{H}_{\mu, p} \triangleq$ $\mathbf{F h}_{\mu}$ in (17). Thus we can conclude that the observation models for the estimation of channel impulse responses $\mathbf{h}_{\mu}$ are

$$
\tilde{\mathbf{Y}}_{\mu, p}=2 \mathbf{F h}_{\mu}+\widetilde{\mathbf{W}}_{\mu, p}, \quad \mu=1,2,
$$

where $\mathbf{F}$ is a $K_{p} \times L$ FFT matrix generated based on pilot indices and $K_{p}$ is the number of pilot symbols per one OFDM block.

Since (18) offers a Bayesian linear model representation, one can obtain a closed-form expression for the MMSE estimation of channel vectors $\mathbf{h}_{1}$ and $\mathbf{h}_{2}$. We should first make the assumptions that impulse responses $\mathbf{h}_{1}$ and $\mathbf{h}_{2}$ are i.i.d. zero-mean complex Gaussian vectors with covariance $\mathbf{C}_{\mathbf{h}}$, and $\mathbf{h}_{1}$ and $\mathbf{h}_{2}$ are independent from $\widetilde{\mathbf{W}}_{1, p} \sim$ $\mathcal{N}\left(\mathbf{0}, \bar{\sigma}^{2} \mathbf{I}_{K_{p}}\right)$ and $\widetilde{\mathbf{W}}_{2, p} \sim \mathcal{N}\left(\mathbf{0}, \bar{\sigma}^{2} \mathbf{I}_{K_{p}}\right)$ and employ PSK pilot symbolassumption to obtain MMSE estimates of $\mathbf{h}_{1}$ and 
$\mathbf{h}_{2}[17]:$

$$
\widehat{\mathbf{h}}_{\mu}=\left(2 \mathbf{F}^{\dagger} \mathbf{F}+\frac{\bar{\sigma}^{2}}{2} \mathbf{C}_{\mathbf{h}}^{-1}\right)^{-1} \mathbf{F}^{\dagger} \tilde{\mathbf{Y}}_{\mu, p}, \quad \mu=1,2 .
$$

Under the assumption that uniformly spaced pilot symbols are inserted with pilot spacing interval $\Delta$ and $K=\Delta \times K_{p}$, correspondingly, $\mathbf{F}^{\dagger} \mathbf{F}$ reduces to $\mathbf{F}^{\dagger} \mathbf{F}=K_{p} \mathbf{I}_{L}$. Then according to (19), and $\mathbf{F}^{\dagger} \mathbf{F}=K_{p} \mathbf{I}_{L}$, we arrive at the expression

$$
\widehat{\mathbf{h}}_{\mu}=\left(2 K_{p} \mathbf{I}_{L}+\frac{\bar{\sigma}^{2}}{2} \mathbf{C}_{\mathbf{h}}^{-1}\right)^{-1} \mathbf{F}^{\dagger} \tilde{\mathbf{Y}}_{\mu, p}, \quad \mu=1,2 .
$$

As it can be seen from (20) MMSE estimation of $\mathbf{h}_{1}$ and $\mathbf{h}_{2}$ for SF-OFDM and ST-OFDM systems still requires the inversion of $\mathbf{C}_{\mathbf{h}}^{-1}$. Therefore it suffers from a high computational complexity. However, it is possible to reduce complexity of the MMSE algorithm by expanding multipath channel as a linear combination of orthogonal basis vectors. The orthogonality of the basis vectors makes the channel representation efficient and mathematically convenient. KL transform which amounts to a generalization of the DFT for random processes can be employed here. This transformation is related to diagonalization of the channel correlation matrix by the unitary eigenvector transformation,

$$
\mathrm{C}_{\mathrm{h}}=\Psi \Lambda \Psi^{\dagger},
$$

where $\Psi=\left[\psi_{0}, \psi_{1}, \ldots, \psi_{L-1}\right], \psi_{l}$ 's are the orthonormal basis vectors, and $\mathbf{g}_{\mu}=\left[g_{\mu, 0}, g_{\mu, 1}, \ldots, g_{\mu, L-1}\right]^{T}$ is zero-mean Gaussian vector with diagonal covariance matrix $\Lambda=E\left\{\mathbf{g}_{\mu} \mathbf{g}_{\mu}^{\dagger}\right\}$.

Thus the vectors $\mathbf{h}_{1}$ and $\mathbf{h}_{2}$ can be expressed as a linear combination of the orthonormal basis vectors, that is, as $\mathbf{h}_{\mu}=\Psi_{\mathbf{g}_{\mu}}$ where $\mu$ is the multipath channel index. As a result, the channel estimation problem in this application is equivalent to estimating the i.i.d. complex Gaussian vectors $\mathbf{g}_{1}$ and $\mathbf{g}_{2}$ which represent KL expansion coefficients for multipath channels $\mathbf{h}_{1}$ and $\mathbf{h}_{2}$.

\subsection{MMSE estimation of $K L$ coefficients}

Substituting $\mathbf{h}_{\mu}=\Psi_{\mathbf{g}_{\mu}}$ in unified observation model (18), we can rewrite it as

$$
\widetilde{\mathbf{Y}}_{\mu, p}=2 \mathbf{F} \Psi \mathbf{g}_{\mu}+\widetilde{\mathbf{W}}_{\mu, p}, \quad \mu=1,2
$$

which is also recognized as a Bayesian linear model, and recall that $\mathbf{g}_{\mu} \sim \mathcal{N}(\mathbf{0}, \boldsymbol{\Lambda})$. As a result, the MMSE estimator of $\mathrm{KL}$ coefficients $\mathbf{g}_{\mu}$ is

$$
\begin{aligned}
\hat{\mathbf{g}}_{\mu} & =\boldsymbol{\Lambda}\left(2 K_{p} \boldsymbol{\Lambda}+\frac{\bar{\sigma}^{2}}{2} \mathbf{I}_{L}\right)^{-1} \boldsymbol{\Psi}^{\dagger} \mathbf{F}^{\dagger} \tilde{\mathbf{Y}}_{\mu, p} \\
& =\boldsymbol{\Gamma} \boldsymbol{\Psi}^{\dagger} \mathbf{F}^{\dagger} \tilde{\mathbf{Y}}_{\mu, p}, \quad \mu=1,2,
\end{aligned}
$$

where

$$
\begin{aligned}
\boldsymbol{\Gamma} & =\boldsymbol{\Lambda}\left(2 K_{p} \boldsymbol{\Lambda}+\frac{\bar{\sigma}^{2}}{2} \mathbf{I}_{L}\right)^{-1} \\
& =\operatorname{diag}\left\{\frac{2 \lambda_{0}}{4 K_{p} \lambda_{0}+\bar{\sigma}^{2}}, \frac{2 \lambda_{1}}{4 K_{p} \lambda_{1}+\bar{\sigma}^{2}}, \ldots, \frac{2 \lambda_{L-1}}{4 K_{p} \lambda_{L-1}+\bar{\sigma}^{2}}\right\}
\end{aligned}
$$

MMSE estimator of $\mathbf{g}$ requires $4 L^{2}+4 L K_{p}+2 L$ real multiplications. From the results presented in [18], ML estimator of $\mathbf{g}_{\mu}$ which requires $4 L^{2}+4 L K_{p}$ real multiplications can be obtained as

$$
\hat{\mathbf{g}}_{\mu}=\frac{1}{2 K_{p}} \boldsymbol{\Psi}^{\dagger} \mathbf{F}^{\dagger} \tilde{\mathbf{Y}}_{\mu, p}, \quad \mu=1,2
$$

It is clear that the complexity of the MMSE estimator in (20) is reduced by the application of KL expansion. However, the complexity of the $\hat{\mathbf{g}}_{\mu}$ can be further reduced by exploiting the optimal truncation property of the KL expansion [15]. A truncated expansion $\mathbf{g}_{\mu_{r}}$ can be formed by selecting $r$ orthonormal basis vectors from all basis vectors that satisfy $\mathbf{C}_{\mathbf{h}} \boldsymbol{\Psi}=\boldsymbol{\Psi} \boldsymbol{\Lambda}$. Thus, a rank- $r$ approximation to $\boldsymbol{\Lambda}_{r}$ is defined as $\boldsymbol{\Lambda}_{r}=\operatorname{diag}\left\{\lambda_{0}, \lambda_{1}, \ldots, \lambda_{r-1}, 0, \ldots, 0\right\}$.

Since the trailing $L-r$ variances $\left\{\lambda_{g_{l}}\right\}_{l=r}^{L-1}$ are small compared to the leading $r$ variances $\left\{\lambda_{g_{l}}\right\}_{l=0}^{r-1}$, the trailing $L-r$ variances are set to zero to produce the approximation. However, typically the pattern of eigenvalues for $\boldsymbol{\Lambda}$ splits the eigenvectors into dominant and subdominant sets. Then the choice of $r$ is more or less obvious. The optimal truncated KL (rank- $r$ ) estimator of (23) now becomes

$$
\hat{\mathbf{g}}_{\mu_{r}}=\boldsymbol{\Gamma}_{r} \boldsymbol{\Psi}^{\dagger} \mathbf{F}^{\dagger} \tilde{\mathbf{Y}}_{\mu, p}
$$

where

$$
\begin{gathered}
\boldsymbol{\Gamma}_{r}=\boldsymbol{\Lambda}_{r}\left(2 K_{p} \boldsymbol{\Lambda}_{r}+\frac{\bar{\sigma}^{2}}{2} \mathbf{I}_{L}\right)^{-1} \\
=\operatorname{diag}\left\{\frac{2 \lambda_{0}}{4 K_{p} \lambda_{0}+\bar{\sigma}^{2}}, \frac{2 \lambda_{1}}{4 K_{p} \lambda_{1}+\bar{\sigma}^{2}}, \ldots,\right. \\
\left.\frac{2 \lambda_{r-1}}{4 K_{p} \lambda_{r-1}+\bar{\sigma}^{2}}, 0, \ldots, 0\right\} .
\end{gathered}
$$

Thus, the truncated MMSE estimator of $\mathbf{g}_{\mu}$ (26) requires $4 L r+4 L K_{p}+2 r$ real multiplications.

\subsection{Estimation of $\mathrm{H}_{\mu, o}(n)$ and $\mathbf{H}_{\mu}(n+1)$}

For the Bayesian MMSE estimation of the channel parameters $\mathbf{H}_{\mu, o}(n)$ and $\mathbf{H}_{\mu}(n+1)$ for SF-OFDM and ST-OFDM, respectively, the unified signal model in (9) can be rewritten by exploiting AR representation in (7) and (8) as

$$
\left[\begin{array}{l}
\mathbf{Y}_{1} \\
\mathbf{Y}_{2}
\end{array}\right]=\frac{1}{\alpha}\left[\begin{array}{cc}
\boldsymbol{X}_{1} & \boldsymbol{X}_{2} \\
-\mathcal{X}_{2}^{\dagger} & \boldsymbol{X}_{1}^{\dagger}
\end{array}\right]\left[\begin{array}{l}
\mathbf{H}_{1^{+}} \\
\mathbf{H}_{2^{+}}
\end{array}\right]+\left[\begin{array}{l}
\mathbf{W}_{1^{+}} \\
\mathbf{W}_{2^{+}}
\end{array}\right] .
$$

The corresponding vectors for SF-OFDM can be listed as

$$
\begin{gathered}
{\left[\frac{\mathbf{H}_{1^{+}}}{\mathbf{H}_{2^{+}}}\right]=\left[\frac{\mathbf{H}_{1, o}(n)}{\mathbf{H}_{2, o}(n)}\right],} \\
{\left[\frac{\mathbf{W}_{1^{+}}}{\mathbf{W}_{2^{+}}}\right]=\left[\frac{\mathbf{W}_{e}(n)-1 / \alpha\left[\mathcal{X}_{e}(n) \boldsymbol{\eta}_{1, o}(n)-\mathcal{X}_{o}(n) \boldsymbol{\eta}_{2, o}(n)\right]}{1 / \alpha \mathbf{W}_{o}(n)}\right] .}
\end{gathered}
$$

and $\lambda_{0}, \lambda_{1}, \ldots, \lambda_{L-1}$ are the singular values of $\Lambda$. 
Moreover for ST-OFDM,

$$
\begin{gathered}
{\left[\frac{\mathbf{H}_{1^{+}}}{\mathbf{H}_{2^{+}}}\right]=\left[\frac{\mathbf{H}_{1}(n+1)}{\mathbf{H}_{2}(n+1)}\right],} \\
{\left[\frac{\mathbf{W}_{1^{+}}}{\mathbf{W}_{2^{+}}}\right]=\left[\frac{\mathbf{W}(n)-(1 / \alpha)\left[\mathcal{X}(n) \boldsymbol{\eta}_{1}(n+1)-\mathcal{X}(n+1) \boldsymbol{\eta}_{2}(n+1)\right]}{(1 / \alpha) \boldsymbol{W}(n+1)}\right] .}
\end{gathered}
$$

Note that $\mathbf{W}_{1^{+}} \sim \mathcal{N}\left(\mathbf{0},\left(\sigma^{2}+2\left(1-|\alpha|^{2}\right) /|\alpha|^{2}\right) \mathbf{I}\right)$ and $\mathbf{W}_{2^{+}} \sim$ $\mathcal{N}\left(\mathbf{0}, \sigma^{2} /|\alpha|^{2} \mathbf{I}\right)$. According to the unified model in (28), corresponding pilot model in (12), and $\mathbf{H}_{\mu^{+}}=\mathbf{F} \Psi \mathbf{g}_{\mu^{+}}$, the observation model becomes

$$
\widetilde{\mathbf{Y}}_{\mu, p}=\frac{2}{\alpha} \mathbf{F} \Psi \mathbf{g}_{\mu^{+}}+\widetilde{\mathbf{W}}_{\mu^{+}, p}, \quad \mu=1,2,
$$

where

$$
\begin{aligned}
& \widetilde{\mathbf{W}}_{1^{+}, p}=X_{1, p}^{\dagger} \mathbf{W}_{1^{+}, p}-X_{2, p} \mathbf{W}_{2^{+}, p}, \\
& \widetilde{\mathbf{W}}_{2^{+}, p}=X_{2, p}^{\dagger} \mathbf{W}_{1^{+}, p}+X_{1, p} \mathbf{W}_{2^{+}, p},
\end{aligned}
$$

and note that $\widetilde{\mathbf{W}}_{\mu^{+}, p} \sim \mathcal{N}\left(\mathbf{0}, \bar{\sigma}^{2} \mathbf{I}\right)$. Thus, the estimation of the $\mathrm{KL}$ coefficient vector $\mathbf{g}_{\mu^{+}}$is

$$
\widehat{\mathbf{g}}_{\mu^{+}}=\widetilde{\Gamma} \Psi^{\dagger} \mathbf{F}^{\dagger} \widetilde{\mathbf{Y}}_{\mu, p}, \quad \mu=1,2,
$$

where

$$
\begin{aligned}
\widetilde{\boldsymbol{\Gamma}}=\Lambda\left(\frac{2}{\alpha^{*}} K_{p} \boldsymbol{\Lambda}+\frac{\alpha}{2} \bar{\sigma}^{2} \mathbf{I}_{L}\right)^{-1} \\
=\operatorname{diag}\left\{\frac{2 \alpha^{*} \lambda_{0}}{4 K_{p} \lambda_{0}+|\alpha|^{2} \bar{\sigma}^{2}}, \frac{2 \alpha^{*} \lambda_{1}}{4 K_{p} \lambda_{1}+|\alpha|^{2} \bar{\sigma}^{2}}, \ldots,\right. \\
\\
\left.\qquad \frac{2 \alpha^{*} \lambda_{L-1}}{4 K_{p} \lambda_{L-1}+|\alpha|^{2} \bar{\sigma}^{2}}\right\} .
\end{aligned}
$$

Note that, choosing $\alpha=1$ results in $\mathbf{H}_{\mu, o}=\mathbf{H}_{\mu, e}$ and $\mathbf{H}_{\mu}(n+1)=\mathbf{H}_{\mu}(n)$, respectively, which significantly simplifies the channel estimation task in transmit diversity OFDM systems.

The performance analysis issues elaborated in the next section only consider the Bayesian MMSE estimator of $\mathbf{g}_{\mu}$ for $\mathbf{H}_{\mu, e}(n)$ and $\mathbf{H}_{\mu}(n)$. However extensions for $\mathbf{g}_{\mu^{+}}$are straightforward.

\section{PERFORMANCE ANALYSIS}

In this section, we turn our attention to analytical performance results. We first exploit the performance of the MMSE channel estimator based on the evaluation of modified Cramer-Rao lower bound, Bayesian MSE together with mismatch analysis. We then derive the closed-form expression for the average SER probability of MRRC.

\subsection{Cramer-Rao lower bound for random $K L$ coefficients}

In this paper, the estimation of unknown random parameters $\mathbf{g}_{\mu}$ is considered via MMSE approach; the modified Fisher information matrix(FIM) therefore needs to be taken into account in the derivation of stochastic CRLB [19]. Fortunately, the modified FIM can be obtained by a straightforward modification of $\mathbf{J}\left(\mathbf{g}_{\mu}\right)$ FIM as

$$
\mathbf{J}_{M}\left(\mathbf{g}_{\mu}\right) \triangleq \mathbf{J}\left(\mathbf{g}_{\mu}\right)+\mathbf{J}_{P}\left(\mathbf{g}_{\mu}\right)
$$

where $\mathbf{J}_{P}\left(\mathbf{g}_{\mu}\right)$ represents the a priori information. Under the assumption that $\mathbf{g}_{\mu}$ and $\widetilde{\mathbf{W}}_{\mu, p}$ are independent of each other and $\widetilde{\mathbf{W}}_{\mu, p}$ is a zero mean, from [19] and (31) the conditional $\mathrm{PDF}$ is given by

$$
\begin{aligned}
p\left(\widetilde{\mathbf{Y}}_{\mu, p} \mid \mathbf{g}_{\mu}\right)= & \frac{1}{\pi^{K_{p}}\left|\mathbf{C}_{\widetilde{\mathbf{W}}_{\mu, p}}\right|} \\
& \times \exp \left\{-\left(\widetilde{\mathbf{Y}}_{\mu, p}-2 \mathbf{F} \Psi \mathbf{g}_{\mu}\right)^{\dagger} \mathbf{C}_{\widetilde{\mathbf{W}}_{\mu, p}^{-1}}^{-1}\right. \\
& \left.\quad \times\left(\widetilde{\mathbf{Y}}_{\mu, p}-2 \mathbf{F} \Psi \mathbf{g}_{\mu}\right)\right\}
\end{aligned}
$$

from which the derivatives follow as

$$
\begin{gathered}
\frac{\partial \ln p\left(\widetilde{\mathbf{Y}}_{\mu, p} \mid \mathbf{g}_{\mu}\right)}{\partial \mathbf{g}_{\mu}^{T}}=2\left(\tilde{\mathbf{Y}}_{\mu, p}-2 \mathbf{F} \Psi \mathbf{g}_{\mu}\right)^{\dagger} \mathbf{C}_{\widetilde{\mathbf{W}}_{\mu, p}^{-1}}^{-1} \mathbf{F}, \\
\frac{\partial^{2} \ln p\left(\widetilde{\mathbf{Y}}_{\mu, p} \mid \mathbf{g}_{\mu}\right)}{\partial \mathbf{g}_{\mu}^{*} \partial \mathbf{g}_{\mu}^{T}}=-4 \boldsymbol{\Psi}^{\dagger} \mathbf{F}^{\dagger} \mathbf{C}_{\widetilde{\mathbf{W}}_{\mu, p}^{-1}}^{-1} \mathbf{F},
\end{gathered}
$$

where the superscript $(\cdot)^{*}$ indicates the conjugation operation. Using $\mathbf{C}_{\widetilde{\mathrm{W}}_{\mu, p}}=\bar{\sigma}^{2} \mathbf{I}_{K_{p}}, \boldsymbol{\Psi}^{\dagger} \boldsymbol{\Psi}=\mathbf{I}_{L}$, and $\mathbf{F}^{\dagger} \mathbf{F}=K_{p} \mathbf{I}_{L}$, and taking the expected value yields the following simple form:

$$
\begin{aligned}
\mathbf{J}\left(\mathbf{g}_{\mu}\right) & =-E\left[\frac{\partial^{2} \ln p\left(\tilde{\mathbf{Y}}_{\mu, p} \mid \mathbf{g}_{\mu}\right)}{\partial \mathbf{g}_{\mu}^{*} \partial \mathbf{g}_{\mu}^{T}}\right] \\
& =-E\left[-\frac{4 K_{p}}{\bar{\sigma}^{2}} \mathbf{I}_{L}\right] \\
& =\frac{4 K_{p}}{\bar{\sigma}^{2}} \mathbf{I}_{L} .
\end{aligned}
$$

Second term in (35) is easily obtained as follows. Consider the prior PDF of $\mathbf{g}_{\mu}(n)$ as

$$
p\left(\mathbf{g}_{\mu}\right)=\frac{1}{\pi^{L}|\boldsymbol{\Lambda}|} \exp \left\{-\mathbf{g}_{\mu}^{\dagger} \boldsymbol{\Lambda}^{-1} \mathbf{g}_{\mu}\right\}
$$

The respective derivatives are found as

$$
\begin{aligned}
& \frac{\partial \ln p\left(\mathbf{g}_{\mu}\right)}{\partial \mathbf{g}_{\mu}^{T}}=-\mathbf{g}_{\mu}^{\dagger} \boldsymbol{\Lambda}^{-1}, \\
& \frac{\partial^{2} \ln p\left(\mathbf{g}_{\mu}\right)}{\partial \mathbf{g}_{\mu}^{*} \partial \mathbf{g}_{\mu}^{T}}=-\boldsymbol{\Lambda}^{-1} .
\end{aligned}
$$

Upon taking the negative expectations, second term in (35) becomes

$$
\begin{aligned}
\mathbf{J}_{P}\left(\mathbf{g}_{\mu}\right) & =-E\left[\frac{\partial^{2} \ln p\left(\mathbf{g}_{\mu}\right)}{\partial \mathbf{g}_{\mu}^{*} \partial \mathbf{g}_{\mu}^{T}}\right] \\
& =-E\left[-\Lambda^{-1}\right] \\
& =\boldsymbol{\Lambda}^{-1}
\end{aligned}
$$


Substituting (38) and (41) in (35) produces for the modified FIM the following:

$$
\begin{aligned}
\mathbf{J}_{M}\left(\mathbf{g}_{\mu}\right) & =\mathbf{J}\left(\mathbf{g}_{\mu}\right)+\mathbf{J}_{P}\left(\mathbf{g}_{\mu}\right) \\
& =\frac{4 K_{p}}{\bar{\sigma}^{2}} \mathbf{I}_{L}+\boldsymbol{\Lambda}^{-1} \\
& =\frac{2}{\bar{\sigma}^{2}}\left(2 K_{p} \mathbf{I}_{L}+\frac{\bar{\sigma}^{2}}{2} \boldsymbol{\Lambda}^{-1}\right) \\
& =\frac{2}{\bar{\sigma}^{2}} \boldsymbol{\Gamma}^{-1} .
\end{aligned}
$$

Inverting the matrix $\mathbf{J}_{M}\left(\mathbf{g}_{\mu}\right)$ yields

$$
\begin{aligned}
\operatorname{CRLB}\left(\hat{\mathbf{g}}_{\mu}\right) & =\mathbf{J}_{M}^{-1}\left(\mathbf{g}_{\mu}\right) \\
& =\frac{\bar{\sigma}^{2}}{2} \boldsymbol{\Gamma} .
\end{aligned}
$$

\subsection{Bayesian MSE}

From the performance of the MMSE estimator for the Bayesian linear model theorem [17], the error covariance matrix is obtained as

$$
\begin{aligned}
\mathbf{C}_{\boldsymbol{\epsilon}_{\mu}} & =\left(\boldsymbol{\Lambda}^{-1}+(2 \mathbf{F} \Psi)^{\dagger} \mathbf{C}_{\widetilde{\mathbf{W}}_{\mu, p}}^{-1}(2 \mathbf{F} \Psi)\right)^{-1} \\
& =\frac{\bar{\sigma}^{2}}{2}\left(2 K_{p} \mathbf{I}_{L}+\frac{\bar{\sigma}^{2}}{2} \boldsymbol{\Lambda}^{-1}\right)^{-1} \\
& =\frac{\bar{\sigma}^{2}}{2} \boldsymbol{\Gamma}
\end{aligned}
$$

Comparing (43) with (44), the error covariance matrix of the MMSE estimator coincides with the stochastic CRLB of the random vector estimator. Thus, $\hat{\mathbf{g}}_{\mu}$ achieves the stochastic CRLB.

We now formalize the Bayesian MSE of the full-rank estimator which is actually an extension of previous evaluation methodology presented in $[20,21]$ :

$$
\begin{aligned}
\mathbf{B}_{\mathrm{MSE}}\left(\hat{\mathbf{g}}_{\mu}\right) & =\frac{1}{L} \operatorname{tr}\left(\mathbf{C}_{\boldsymbol{\epsilon}_{\mu}}\right) \\
& =\frac{1}{L} \operatorname{tr}\left(\frac{\bar{\sigma}^{2}}{2} \boldsymbol{\Gamma}\right)=\frac{1}{L} \sum_{i=0}^{L-1} \frac{\bar{\sigma}^{2} \lambda_{i}}{\bar{\sigma}^{2}+4 K_{p} \lambda_{i}},
\end{aligned}
$$

where, substituting $\sigma^{2}=1 / \mathrm{SNR}$ in $\bar{\sigma}^{2}, \bar{\sigma}^{2}=1+|\alpha|^{2} /$ $|\alpha|^{2}$ SNR $+2\left(1-|\alpha|^{2}\right) /|\alpha|^{2}$, and tr denotes trace operator on matrices.

Following the results presented in $[20,21], \mathbf{B}_{\mathrm{MSE}}\left(\hat{\mathbf{g}}_{\mu}\right)$ given in (45) can also be computed for the truncated (lowrank) case as follows:

$$
\mathbf{B}_{\mathrm{MSE}}\left(\hat{\mathbf{g}}_{\mu_{r}}\right)=\frac{1}{L} \sum_{i=0}^{r-1} \frac{\bar{\sigma}^{2} \lambda_{i}}{\bar{\sigma}^{2}+4 K_{p} \lambda_{i}}+\frac{1}{L} \sum_{i=r}^{L-1} \lambda_{i} .
$$

Notice that the second term in (46) is the sum of the powers in the KL transform coefficients not used in the truncated estimator. Thus, truncated $\mathbf{B}_{\mathrm{MSE}}\left(\hat{\mathbf{g}}_{\mu_{r}}\right)$ can be lower bounded by $(1 / L) \sum_{i=r}^{L-1} \lambda_{i}$ which will cause an irreducible error floor in the SER results.

\subsection{Mismatch analysis}

In mobile wireless communications, the channel statistics depend on the particular environment, for example, indoor or outdoor, urban or suburban, and change with time. Hence, it is important to analyze the performance degradation due to a mismatch of the estimator with respect to the channel statistics as well as the SNR, and to study the choice of the channel correlation and SNR for this estimator so that it is robust to variations in the channel statistics. As a performance measure, we use Bayesian MSE (45).

In practice, the true channel correlations and SNR are not known. If the MMSE channel estimator is designed to match the correlation of a multipath channel impulse response $\mathbf{C}_{\mathbf{h}}$ and SNR, but the true channel parameters $\widetilde{\mathbf{h}}_{\mu}$ have the correlation $\mathbf{C}_{\tilde{\mathbf{h}}}$ and the true $\overparen{\mathrm{SNR}}$, then average Bayesian MSE for the designed channel estimator is extended from [21] as follows

(i) SNR mismatch:

$$
\mathbf{B}_{\mathrm{MSE}}\left(\widehat{\mathbf{g}_{\mu}}\right)=\frac{1}{L} \sum_{i=0}^{L-1} \lambda_{i} \tilde{\sigma}^{2} \frac{4 K_{p} \lambda_{i}+\bar{\sigma}^{4} / \tilde{\sigma}^{2}}{\left(4 K_{p} \lambda_{i}+\bar{\sigma}^{2}\right)^{2}}
$$

where

$$
\begin{aligned}
& \bar{\sigma}^{2}=\frac{1+|\alpha|^{2}}{|\alpha|^{2} \mathrm{SNR}}+\frac{2\left(1-|\alpha|^{2}\right)}{|\alpha|^{2}}, \\
& \tilde{\sigma}^{2}=\frac{1+|\alpha|^{2}}{|\alpha|^{2} \widetilde{\mathrm{SNR}}}+\frac{2\left(1-|\alpha|^{2}\right)}{|\alpha|^{2}} .
\end{aligned}
$$

(ii) Correlation mismatch:

$$
\mathbf{B}_{\mathrm{MSE}}\left(\hat{\mathbf{g}}_{\mu}\right)=\frac{1}{L} \sum_{i=0}^{L-1} \frac{\tilde{\lambda}_{i} \bar{\sigma}^{2}+4 K_{p} \lambda_{i}\left(\tilde{\lambda}_{i}+\lambda_{i}-2 \beta_{i}\right)}{\bar{\sigma}^{2}+4 K_{p} \lambda_{i}},
$$

where $\tilde{\lambda}_{i}$ is the $i$ th diagonal element of $\tilde{\Lambda}=\Psi^{\dagger} \mathbf{C}_{\widetilde{\mathbf{h}}} \boldsymbol{\Psi}$, and $\beta_{i}$ is $i$ th diagonal element of the real part of the crosscorrelation matrix between $\tilde{\mathbf{g}}_{\mu}$ and $\mathbf{g}_{\mu}$.

\subsection{Theoretical SER for SF/ST-OFDM systems}

Let us define $\overline{\mathbf{Y}}=\left[\begin{array}{ll}\mathbf{Y}_{1} & \mathbf{Y}_{2}^{*}\end{array}\right]^{T}$ and cast (9) in a matrix/vector form:

$$
\underbrace{\left[\begin{array}{c}
\mathbf{Y}_{1} \\
\mathbf{Y}_{2}^{*}
\end{array}\right]}_{\overline{\mathbf{Y}}}=\underbrace{\left[\begin{array}{ll}
\mathscr{H}_{1} & \mathscr{H}_{2} \\
\mathscr{H}_{2}^{\dagger} & -\mathscr{H}_{1}^{\dagger}
\end{array}\right]}_{\overline{\mathscr{H}}} \underbrace{\left[\begin{array}{l}
\mathbf{X}_{1} \\
\mathbf{X}_{2}
\end{array}\right]}_{\mathbf{X}}+\underbrace{\left[\begin{array}{l}
\mathbf{W}_{1} \\
\mathbf{W}_{2}^{*}
\end{array}\right]}_{\overline{\mathbf{W}}},
$$

where $\mathscr{H}_{\mu}=\operatorname{diag}\left(\mathbf{H}_{\mu}\right)$. By premultiplying (50) by $\overline{\mathscr{H}}^{\dagger}$ the signal model for maximal ratio receive combiner (MRRC) can be obtained as

$$
\begin{aligned}
{\left[\begin{array}{c}
\breve{\mathbf{Y}}_{1} \\
\breve{\mathbf{Y}}_{2}
\end{array}\right]=} & {\left[\begin{array}{cc}
\left\|\mathcal{H}_{1}\right\|^{2}+\left\|\mathscr{H}_{2}\right\|^{2} & 0 \\
0 & \left\|\mathscr{H}_{1}\right\|^{2}+\left\|\mathscr{H}_{2}\right\|^{2}
\end{array}\right] } \\
& \times\left[\begin{array}{l}
\mathbf{X}_{1} \\
\mathbf{X}_{2}
\end{array}\right]+\left[\begin{array}{c}
\breve{\mathbf{W}}_{1} \\
\breve{\mathbf{W}}_{2},
\end{array}\right],
\end{aligned}
$$


where

$$
\begin{aligned}
\breve{\mathbf{Y}}_{1} & =\mathscr{H}_{1}^{\dagger} \mathbf{Y}_{1}+\mathscr{H}_{2} \mathbf{Y}_{2}^{*}, \\
\breve{\mathbf{Y}}_{2} & =\mathscr{H}_{2}^{\dagger} \mathbf{Y}_{1}-\mathscr{H}_{1} \mathbf{Y}_{2}^{*}, \\
\breve{\mathbf{W}}_{1} & =\mathscr{H}_{1}^{\dagger} \mathbf{W}_{1}+\mathscr{H}_{2} \mathbf{W}_{2}^{*}, \\
\breve{\mathbf{W}}_{2} & =\mathscr{H}_{2}^{\dagger} \mathbf{W}_{1}-\mathscr{H}_{1} \mathbf{W}_{2}^{*} .
\end{aligned}
$$
nel is

Thus, at the output of MRRC the signal for $k$ th subchan-

$$
\breve{\mathbf{Y}}_{\mu}(k)=\left(\left|\mathbf{H}_{1}(k)\right|^{2}+\left|\mathbf{H}_{2}(k)\right|^{2}\right) \mathbf{X}_{\mu}(k)+\breve{\mathbf{W}}_{\mu}(k) .
$$

Assuming that $\mathbf{H}_{\mu}(k)=\rho_{\mu} e^{-j \theta_{\mu}},\left(\breve{\mathbf{W}}_{\mu}(k) \mid \rho_{1}, \rho_{2}, \theta_{1}, \theta_{2}\right) \sim$ $\mathcal{N}\left(0, \breve{\sigma}^{2}\right)$, where $\breve{\sigma}^{2}=\left(\rho_{1}^{2}+\rho_{2}^{2}\right) \sigma^{2}$, and the faded signal energy at MRRC $\breve{E}_{s}=\left(\rho_{1}^{2}+\rho_{2}^{2}\right)^{2} E_{s}$. Thus, the symbol error probability of QPSK for given $\rho_{1}, \rho_{2}, \theta_{1}, \theta_{2}$ is

$$
\begin{aligned}
\operatorname{Pr}\left(e \mid \rho_{1}, \rho_{2}, \theta_{1}, \theta_{2}\right) & \\
= & 2 Q\left(\sqrt{\frac{\breve{E}_{s}}{\breve{\sigma}^{2}}}\right)-Q^{2}\left(\sqrt{\frac{\breve{E}_{s}}{\breve{\sigma}^{2}}}\right) \\
& =2 Q\left(\sqrt{\left(\rho_{1}^{2}+\rho_{2}^{2}\right) \frac{E_{s}}{\sigma^{2}}}\right)-Q^{2}\left(\sqrt{\left(\rho_{1}^{2}+\rho_{2}^{2}\right) \frac{E_{s}}{\sigma^{2}}}\right) \\
& =2 Q\left(\sqrt{\left(\rho_{1}^{2}+\rho_{2}^{2}\right) \mathrm{SNR}}\right)-Q^{2}\left(\sqrt{\left(\rho_{1}^{2}+\rho_{2}^{2}\right) \mathrm{SNR}}\right) .
\end{aligned}
$$

Bearing in mind that $\operatorname{Pr}\left(e \mid \rho_{1}, \rho_{2}, \theta_{1}, \theta_{2}\right)$ does not depend on $\theta_{1}$ and $\theta_{2}$, note that

$$
\begin{aligned}
\operatorname{Pr}\left(e \mid \rho_{1}, \rho_{2}\right) \\
\quad=\iint_{-\pi}^{\pi} \operatorname{Pr}\left(e, \theta_{1}, \theta_{2} \mid \rho_{1}, \rho_{2}\right) d \theta_{2} d \theta_{1} \\
=\iint_{-\pi}^{\pi} \operatorname{Pr}\left(e \mid \rho_{1}, \rho_{2}, \theta_{1}, \theta_{2}\right) p\left(\theta_{1}\right) p\left(\theta_{2}\right) d \theta_{2} d \theta_{1} \\
=\operatorname{Pr}\left(e \mid \rho_{1}, \rho_{2}, \theta_{1}, \theta_{2}\right) \iint_{-\pi}^{\pi} p\left(\theta_{1}\right) p\left(\theta_{2}\right) d \theta_{2} d \theta_{1} \\
=\operatorname{Pr}\left(e \mid \rho_{1}, \rho_{2}, \theta_{1}, \theta_{2}\right) .
\end{aligned}
$$

We then substitute (55) in the following equation:

$$
\begin{aligned}
& \operatorname{Pr}(e)= \iint_{0}^{\infty} \iint_{-\pi}^{\pi} p\left(\rho_{1}, \rho_{2}, \theta_{1}, \theta_{2}\right) \\
& \times \operatorname{Pr}\left(e \mid \rho_{1}, \rho_{2}, \theta_{1}, \theta_{2}\right) d \theta_{2} d \theta_{1} d \rho_{2} d \rho_{1} \\
&= \iint_{0}^{\infty} \iint_{-\pi}^{\pi} p\left(\rho_{1}, \rho_{2}, \theta_{1}, \theta_{2}\right) \\
& \quad \times \operatorname{Pr}\left(e \mid \rho_{1}, \rho_{2}\right) d \theta_{2} d \theta_{1} d \rho_{2} d \rho_{1} \\
&=\iint_{0}^{\infty} p\left(\rho_{1}, \rho_{2}\right) \operatorname{Pr}\left(e \mid \rho_{1}, \rho_{2}\right) d \rho_{2} d \rho_{1} .
\end{aligned}
$$

Since channels $\mathbf{H}_{1}$ and $\mathbf{H}_{2}$ are independent, $\rho_{1}$ and $\rho_{2}$ are also independent, $p\left(\rho_{1}, \rho_{2}\right)=p\left(\rho_{1}\right) p\left(\rho_{2}\right)$. Thus (56) takes the following form:

$$
\begin{aligned}
\operatorname{Pr}(e)= & \iint_{0}^{\infty} p\left(\rho_{1}\right) p\left(\rho_{2}\right) \operatorname{Pr}\left(e \mid \rho_{1}, \rho_{2}\right) d \rho_{2} d \rho_{1} \\
= & \iint_{0}^{\infty} 4 \rho_{1} \rho_{2} e^{-\left(\rho_{1}^{2}+\rho_{2}^{2}\right)} \\
\quad & \left(2 Q \left(\sqrt{\left.\left(\rho_{1}^{2}+\rho_{2}^{2}\right) \mathrm{SNR}\right)}\right.\right. \\
& \left.-Q^{2}\left(\sqrt{\left(\rho_{1}^{2}+\rho_{2}^{2}\right) \mathrm{SNR}}\right)\right) d \rho_{2} d \rho_{1} .
\end{aligned}
$$

If we now apply $\rho_{1}=\zeta \cos (\alpha)$ and $\rho_{2}=\zeta \sin (\alpha)$ transformations, we arrive at the following SER expression for STOFDM and SF-OFDM systems:

$$
\begin{aligned}
\operatorname{Pr}(e)= & \int_{0}^{\infty} \int_{0}^{\pi / 2} 2 \zeta^{3} \sin (2 \alpha) e^{-\zeta^{2}} \\
& \times\left(2 Q\left(\sqrt{\zeta^{2} \mathrm{SNR}}\right)-Q^{2}\left(\sqrt{\zeta^{2} \mathrm{SNR}}\right)\right) d \alpha d \zeta \\
= & \int_{0}^{\infty} 2 \zeta^{3} e^{-\zeta^{2}}\left(2 Q\left(\sqrt{\zeta^{2} \mathrm{SNR}}\right)-Q^{2}\left(\sqrt{\zeta^{2} \mathrm{SNR}}\right)\right) d \zeta \\
= & \frac{3}{4}-\left(\frac{1}{2}+\frac{1}{\pi} \arctan \left(\gamma_{2}\right)\right) \gamma_{2}^{3} \gamma_{3}-\gamma_{2}^{2} \gamma_{1}
\end{aligned}
$$

or by neglecting the $Q^{2}(\cdot)$ term in (58) we get simplified form as

$$
\operatorname{Pr}(e)=1-\gamma_{2}^{3} \gamma_{3}
$$

where

$$
\begin{aligned}
\gamma_{1} & =\frac{1}{2 \pi(\mathrm{SNR}+1)}, \\
\gamma_{2} & =\sqrt{\frac{\mathrm{SNR}}{\mathrm{SNR}+2}} \\
\gamma_{3} & =\frac{\mathrm{SNR}+3}{\mathrm{SNR}} .
\end{aligned}
$$

\section{SIMULATIONS}

In this section, we investigate the performance of the pilot-aided MMSE channel estimation algorithm proposed for both SF-OFDM and ST-OFDM systems. The diversity scheme with two transmit and one receive antenna is considered. Channel impulse responses $\mathbf{h}_{\mu}$ are generated according to $\mathbf{C}_{\mathbf{h}}=\left(1 / K^{2}\right) \mathcal{F}^{\dagger} \mathbf{C}_{\mathbf{H}} \mathcal{F}$ where $\mathbf{C}_{\mathbf{H}}$ is the covariance matrix of the doubly-selective fading channel model. In this model, $\mathbf{H}_{\mu}(k)$ 's are with an exponentially decaying power-delay profile $\theta\left(\tau_{\mu}\right)=C \exp \left(-\tau_{\mu} / \tau_{\text {rms }}\right)$ and delays $\tau_{\mu}$ that are uniformly and independently distributed over the length of the cyclic prefix. $C$ is a normalizing constant. Note that the normalized discrete channel correlations for different subcarriers and blocks of this channel model were presented in [3] as follows:

$$
\begin{gathered}
c_{f}\left(k, k^{\prime}\right) \\
=\frac{1-\exp \left[-L\left[1 / \tau_{\mathrm{rms}}+2 \pi j\left(k-k^{\prime}\right) / K\right)\right]}{\tau_{\mathrm{rms}}\left(1-\exp \left(-L / \tau_{\mathrm{rms}}\right)\right)\left(1 / \tau_{\mathrm{rms}}+2 \pi j\left(k-k^{\prime}\right) / K\right)}, \\
c_{t}\left(n, n^{\prime}\right)=J_{o}\left(2 \pi\left(n-n^{\prime}\right) f_{d} T_{s}\right),
\end{gathered}
$$

where $J_{o}$ is the zeroth-order Bessel function of the first kind and $f_{d}$ is the Doppler frequency.

The scenario for SF-OFDM simulation study consists of a wireless QPSK OFDM system. The system has a $2.344 \mathrm{MHz}$ bandwidth (for the pulse roll-off factor $a=0.2$ ) and is divided into 512 tones with a total period of 136 microseconds, of which 5.12 microseconds constitute the cyclix prefix $(L=$ 20). The uncoded data rate is $7.813 \mathrm{Mbits} / \mathrm{s}$. We assume that 


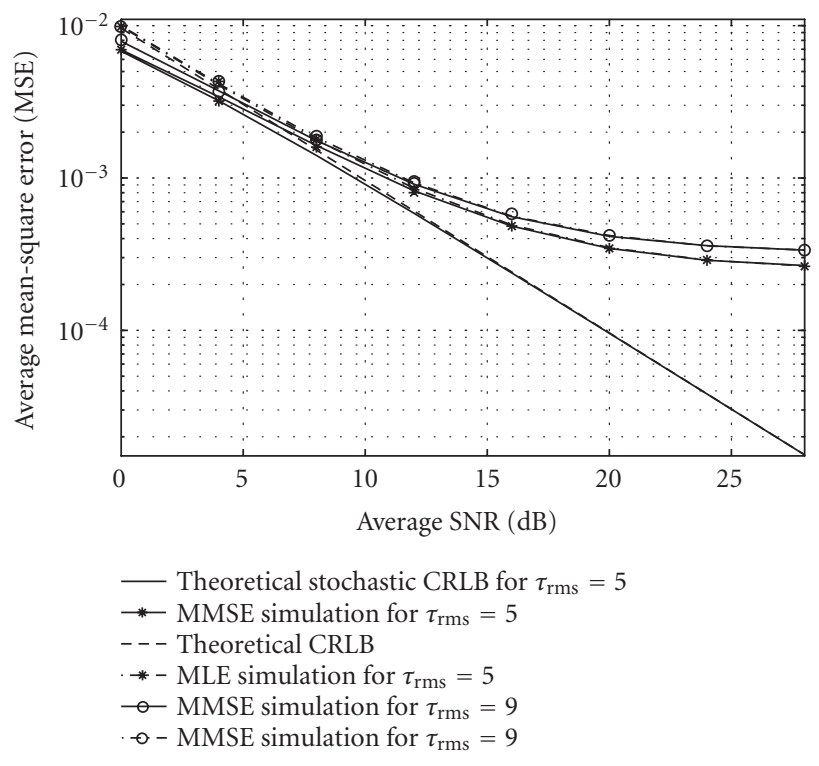

FIgURE 3: Performance of the proposed MMSE and MLE together with BMSE and CRLB for ST-OFDM.

the rms width is $\tau_{\text {rms }}=5$ samples ( 1.28 microseconds) for the power-delay profile. Keeping the transmission efficiency $3.333 \mathrm{bits} / \mathrm{s} / \mathrm{Hz}$ fixed, we also simulate ST-OFDM system.

\subsection{Mean-square-error performance of the channel estimation}

The proposed MMSE channel estimators of (23) are implemented for both SF-OFDM and ST-OFDM, and compared in terms of average Bayesian MSE for a wide range of signal-tonoise ratio (SNR) levels. Average Bayesian mean-square error(BMSE) is defined as the norm of the difference between the vectors $\mathbf{g}=\left[\mathbf{g}_{1}^{T}, \mathbf{g}_{2}^{T}\right]^{T}$ and $\hat{\mathbf{g}}$, representing the true and the estimated values of channel parameters, respectively. Namely,

$$
\mathrm{MSE}=\frac{1}{2 L}\|\mathbf{g}-\hat{\mathbf{g}}\|^{2}
$$

\subsection{MMSE approach}

We use a pilot symbol for every ten $(\Delta=10)$ symbols. The MSE at each SNR point is averaged over 10000 realizations. We compare the experimental MSE performance and its theoretical Bayesian MSE of the proposed full-rank MMSE estimator with maximum likelihood (ML) estimator and its corresponding Cramer-Rao lower bound (CRLB) for SF and ST-OFDM systems. Figures 3 and 4 confirm that MMSE estimator performs better than ML estimator at low SNR. However, the two approaches have comparable performance at high SNRs. To observe the performance, we also present the MMSE and ML estimated channel SER results together with theoretical SER in Figures 5 and 6. Due to the fact that spaces between the pilot symbols are not chosen as a factor of the number of subcarriers, an error floor is observed in Figures $3,4,5$, and 6 . In the case of choosing the pilot space as a factor of number of subcarriers, the error floor vanishes because of

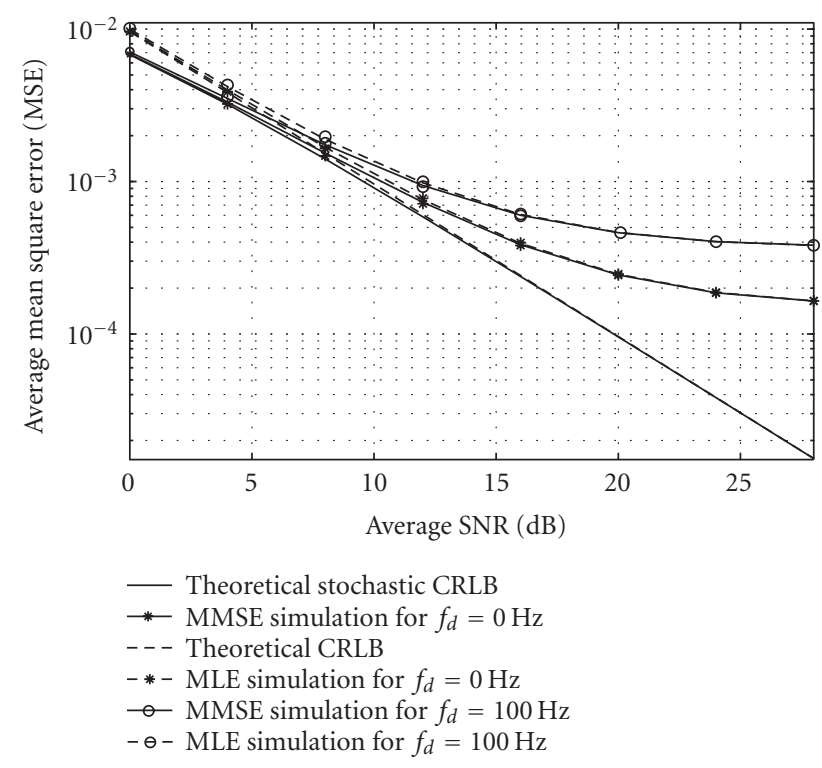

FIGURE 4: Performance of the proposed MMSE and MLE together with BMSE and CRLB for ST-OFDM.

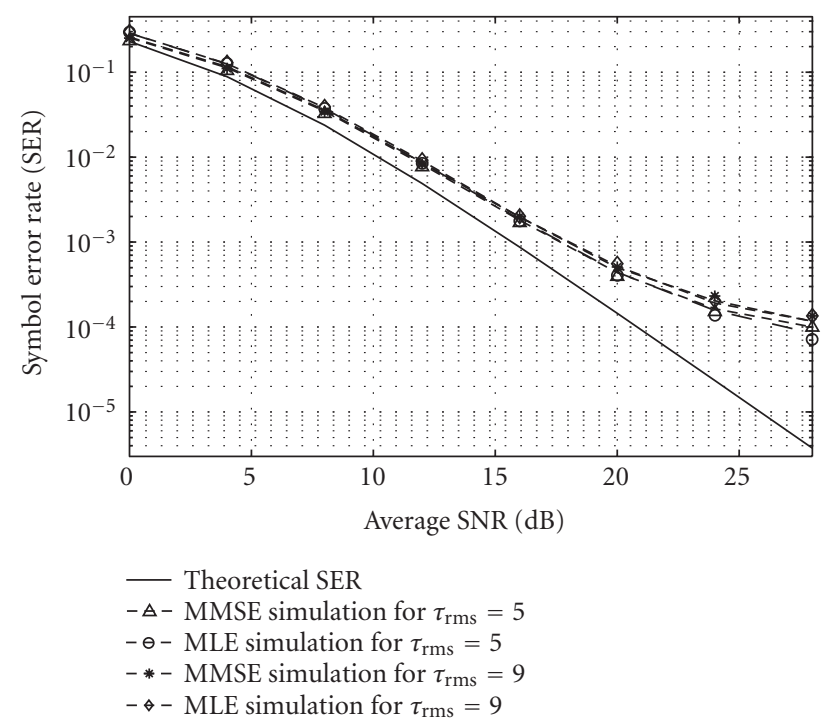

FIGURE 5: Symbol error rate results for SF-OFDM.

the fact that the orthogonality condition between the subcarriers at pilot locations is satisfied. In other words, the curves labeled as simulation results for MMSE estimator and ML estimator fit to the theoretical curve at high SNRs. It also shows that the MMSE-estimated channel SER results are better than ML-estimated channel SER especially at low SNR.

\section{SNR design mismatch}

In order to evaluate the performance of the proposed fullrank MMSE estimator to mismatch only in SNR design, the estimator is tested when SNRs of 10 and $30 \mathrm{~dB}$ are used in the design. The MSE curves for a design SNR of 10, $30 \mathrm{~dB}$ are 


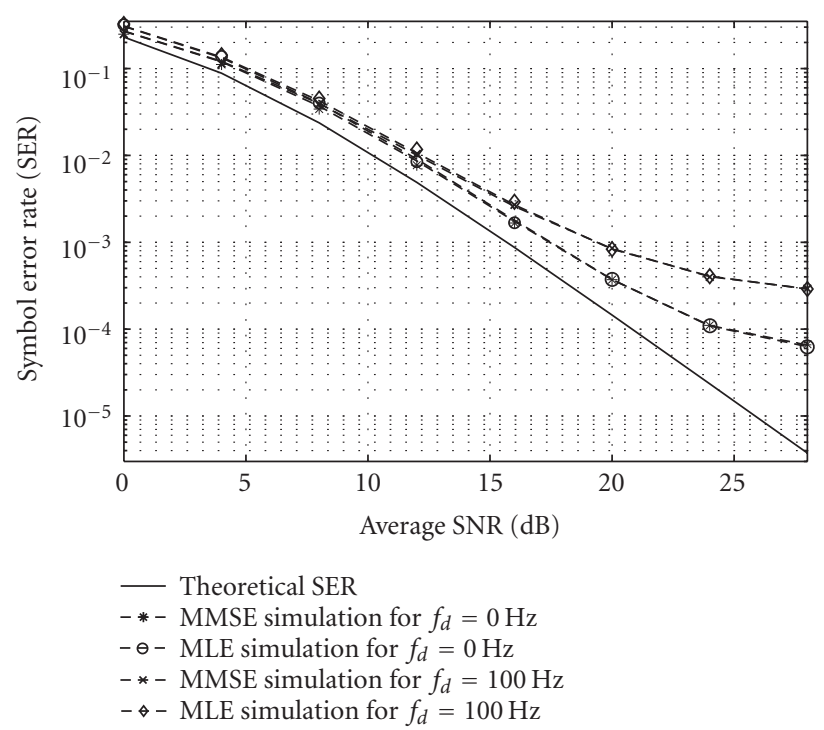

FIGURE 6: Symbol error rate results for ST-OFDM.

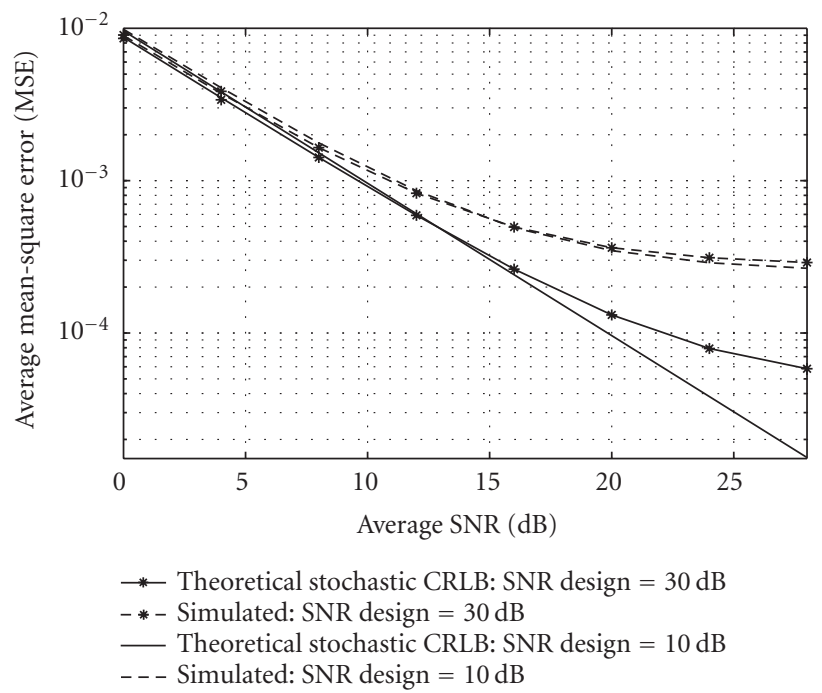

Figure 7: Effects of SNR mismatch on MSE for SF-OFDM.

shown in Figures 7 and 8 . The performance of the MMSE estimator for high-SNR $(30 \mathrm{~dB})$ design is better than low-SNR $(10 \mathrm{~dB})$ design across a range of SNR values $(0-28 \mathrm{~dB})$. This result confirms that channel estimation error is concealed in noise for low-SNR whereas it tends to dominate for highSNR. Thus, the system performance degrades especially for low-SNR design.

\section{Correlation mismatch}

To analyze full-rank MMSE estimator's performance further, we need to study sensitivity of the estimator to design errors, that is, correlation mismatch. We therefore designed the estimator for a uniform channel correlation which gives the worst MSE performance among all channels $[20,22]$ and

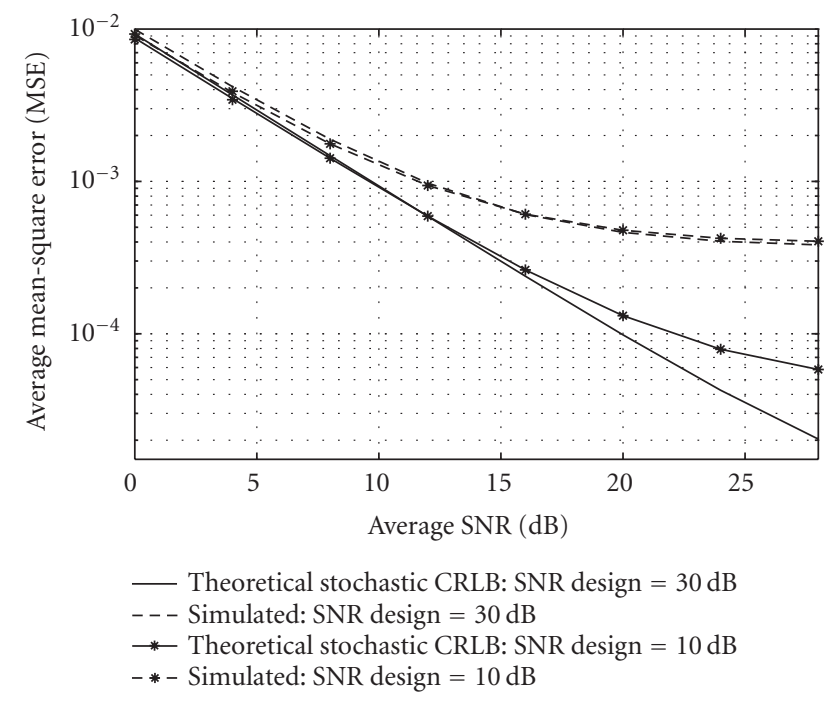

FIGURE 8: Effects of SNR mismatch on MSE for ST-OFDM $\left(f_{d}=\right.$ $100 \mathrm{~Hz})$.

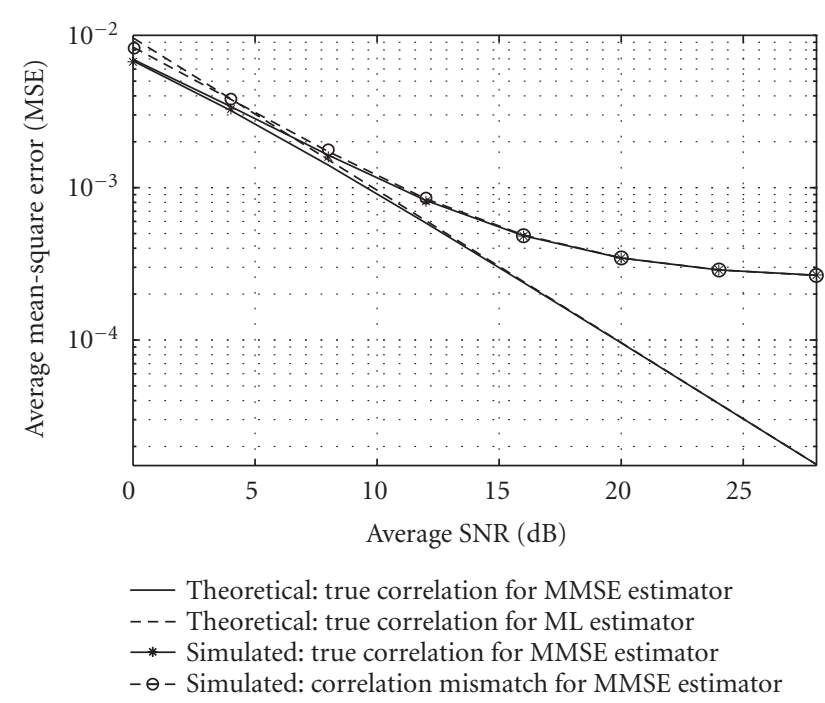

FIGURE 9: Effects of correlation mismatch on MSE for SF-OFDM.

evaluated for an exponentially decaying power-delay profile. The uniform channel correlation between the attenuations can be obtained by letting $\tau_{\text {rms }} \rightarrow \infty$ in (61), resulting in

$$
c_{f}(k)=\frac{1-\exp (2 \pi j L k / K)}{2 \pi j k / K} .
$$

Figures 9 and 10 demonstrate the estimator's sensitivity to the channel statistics in terms of average MSE performance measure. As can be seen from Figures 9 and 10 only small performance loss is observed for low SNRs when the estimator is designed for mismatched channel statistics. This justifies the result that a design for worst correlation is robust to mismatch. 


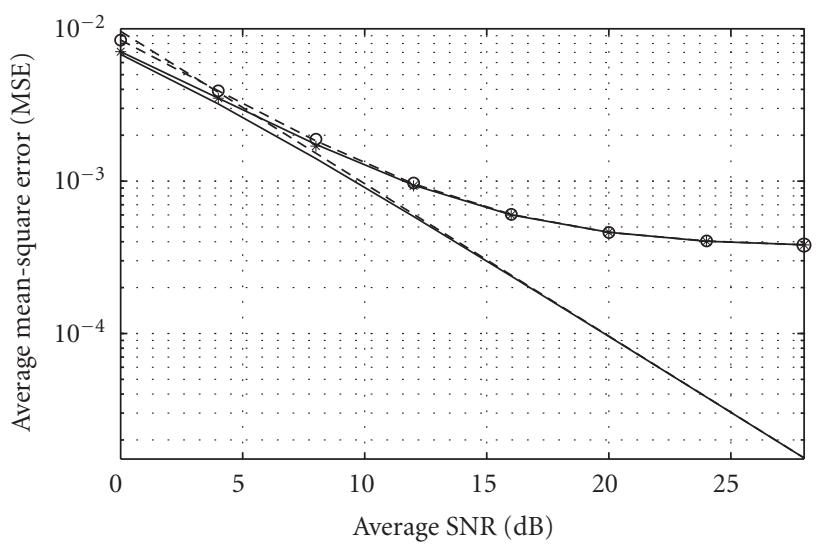

- Theoretical: true correlation for MMSE estimator

- - - Theoretical: true correlation for ML estimator

* Simulated: true correlation for MMSE estimator

$-\theta$ - Simulated: correlation mismatch for MMSE estimator

FIGURE 10: Effects of correlation mismatch on MSE for ST-OFDM $\left(f_{d}=100 \mathrm{~Hz}\right)$.

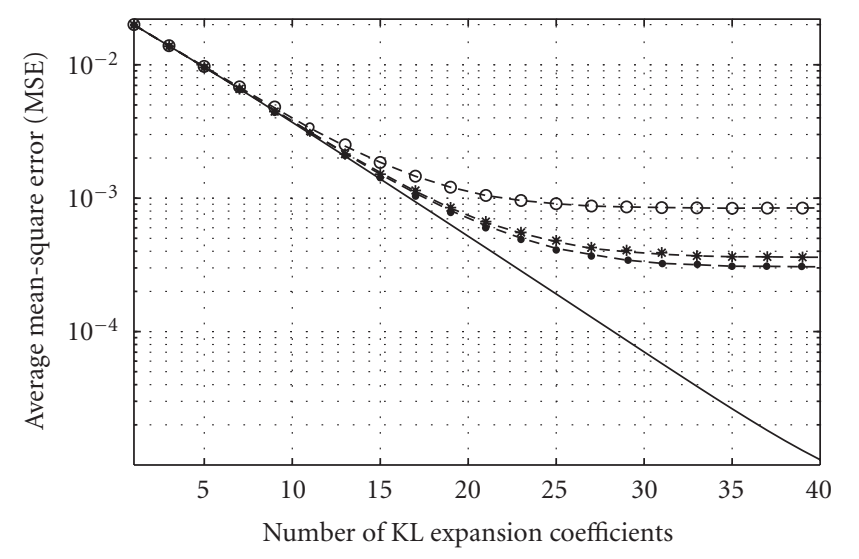

- Theoretical stochastic MSE: SNR $=30 \mathrm{~dB}$
- - Simulated: $\mathrm{SNR}=30 \mathrm{~dB}$
*- Simulated: SNR $=20 \mathrm{~dB}$
$-\Theta$ - Simulated: SNR $=10 \mathrm{~dB}$

FIGURE 11: MSE as a function of KL expansion coefficients for SFOFDM.

\section{Performance of the truncated estimator}

The truncated estimator performance is also studied as a function of the number of KL coefficients. Figures 11 and 12 are plotted for $L=40, \tau_{\mathrm{rms}}=5$ samples and $L=40, f_{d}=$ $100 \mathrm{~Hz}$, respectively. Figures 11 and 12 present the MSE result of the truncated MMSE estimator for $\mathrm{SNR}=10,20$, and $30 \mathrm{~dB}$. If only a few expansion coefficients are employed to reduce the complexity of the proposed estimator, then the MSE between channel parameters becomes large. However, if the number of parameters in the expansion is increased, the irreducible error floor still occurs.

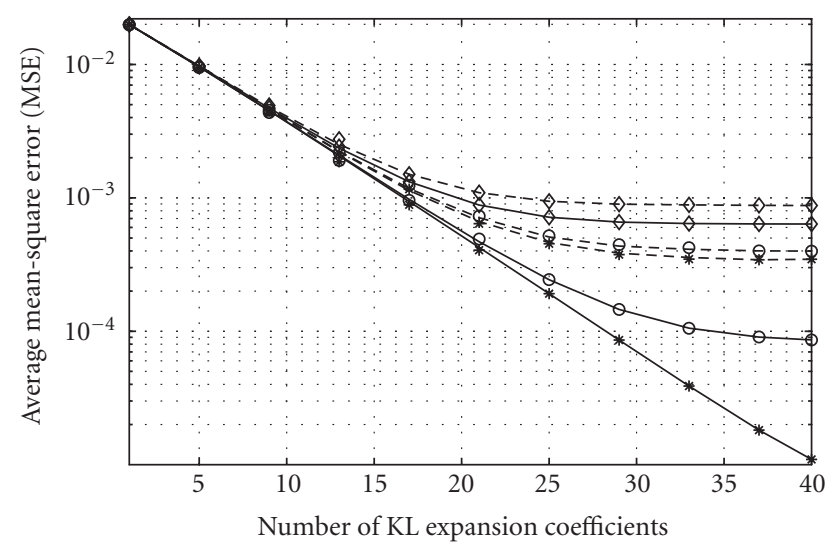

$\rightarrow$ Theoretical stochastic CRLB: SNR $=10 \mathrm{~dB}$
$-\diamond-$ Simulated: SNR $=10 \mathrm{~dB}$
$\rightarrow$ Theoretical stochastic CRLB: SNR $=20 \mathrm{~dB}$
$-\bullet-$ Simulated: SNR $=20 \mathrm{~dB}$
$\rightarrow-$ Theoretical stochastic CRLB: SNR $=30 \mathrm{~dB}$
$-*-$ Simulated: SNR $=30 \mathrm{~dB}$

FIGURE 12: MSE as a function of KL expansion coefficients for ST$\operatorname{OFDM}\left(f_{d}=100 \mathrm{~Hz}\right)$.

\section{CONCLUSION}

We consider the design of low-complexity MMSE channel estimators for SF/ST-OFDM systems in unknown wireless dispersive fading channels. We first derive the MMSE estimator based on the stochastic orthogonal expansion representation of the channel via KL transform. Based on such representation, we show that no matrix inversion is needed in the MMSE algorithm. Therefore, the computational cost for implementing the proposed MMSE estimator is low and computation is numerically stable. Moreover, the performance of our proposed method was first studied through the derivation of stochastic CRLB for Bayesian approach. Since the actual channel statistics and SNR may vary within OFDM block, we have also analyzed the effect of modelling mismatch on the estimator performance and shown both analytically and through simulations that the performance degradation due to such mismatch is negligible for low-SNR values. Obvious directions for future work include developing sequential MMSE, Kalman filtering, and sequential MonteCarlo-based approaches to track channel variations.

\section{ACKNOWLEDGMENTS}

This research has been conducted within the NEWCOM Network of Excellence in Wireless Communications funded through the EC $6^{\text {th }}$ Framework Programme. The present work was also supported in part by the Research Fund of Istanbul University Project numbers BYP-938/02032006, and UDP-599/28072005, UDP-582/12072005 and by The Scientific and Technological Research Council of Turkey (TÜBİTAK) under Grant 104E166.

\section{REFERENCES}

[1] R. Van Nee and R. Prasad, OFDM Wireless Multimedia Communications, Artech House, London, UK, 2000. 
[2] H. Sari, G. Karam, and I. Jeanclaude, "Transmission techniques for digital terrestrial TV broadcasting," IEEE Communications Magazine, vol. 33, no. 2, pp. 100-109, 1995.

[3] B. Yang, Z. Cao, and K. B. Letaief, "Analysis of low-complexity windowed DFT-based MMSE channel estimator for OFDM systems," IEEE Transactions on Communications, vol. 49, no. 11, pp. 1977-1987, 2001.

[4] Y. (G.) Li, N. Seshadri, and S. Ariyavisitakul, "Channel estimation for OFDM systems with transmitter diversity in mobile wireless channels," IEEE Journal on Selected Areas in Communications, vol. 17, no. 3, pp. 461-471, 1999.

[5] Y. (G.) Li, "Simplified channel estimation for OFDM systems with multiple transmit antennas," IEEE Transactions on Wireless Communications, vol. 1, no. 1, pp. 67-75, 2002.

[6] K. F. Lee and D. B. Williams, "A space-frequency transmitter diversity technique for OFDM systems," in Proceedings of IEEE Global Telecommunications Conference, vol. 3, pp. 1473-1477, San Francisco, Calif, USA, November 2000.

[7] Z. Liu, Y. Xin, and G. B. Giannakis, "Space-time-frequency coded OFDM over frequency-selective fading channels," IEEE Transactions on Signal Processing, vol. 50, no. 10, pp. 24652476, 2002.

[8] H. Bolcskei and A. J. Paulraj, "Space-frequency coded broadband OFDM systems," in Proceedings of IEEE Wireless Communications and Networking Conference (WCNC '00), vol. 1, pp. 1-6, Chicago, Ill, USA, September 2000.

[9] S. M. Alamouti, "A simple transmit diversity technique for wireless communications," IEEE Journal on Selected Areas in Communications, vol. 16, no. 8, pp. 1451-1458, 1998.

[10] V. Tarokh, N. Seshadri, and A. R. Calderbank, "Space-time codes for high data rate wireless communication: performance criterion and code construction," IEEE Transactions on Information Theory, vol. 44, no. 2, pp. 744-765, 1998.

[11] V. Tarokh, H. Jafarkhani, and A. R. Calderbank, "Space-time block codes from orthogonal designs," IEEE Transactions on Information Theory, vol. 45, no. 5, pp. 1456-1467, 1999.

[12] I. Tolochko and M. Faulkner, "Real time LMMSE channel estimation for wireless OFDM systems with transmitter diversity," in Proceedings of IEEE 56th Vehicular Technology Conference (VTC'02), vol. 3, pp. 1555-1559, Vancouver, BC, Canada, September 2002.

[13] X. Hou, Y. Xu, B. Zheng, and H. Luo, "A time-domain approach for channel estimation in MIMO-OFDM-based wireless networks," IEICE Transactions on Communications, vol. E88-B, no. 1, pp. 3-9, 2005.

[14] H. Miao and M. J. Juntti, "Data aided channel estimation for wireless MIMO-OFDM systems," in Multicarrier Spread Spectrum, K. Fazel and S. Kaiser, Eds., pp. 211-218, Kluwer Academic, Boston, Mass, USA, 2004.

[15] K.-W. Yip and T.-S. Ng, "Karhunen-Loève expansion of the WSSUS channel output and its application to efficient simulation," IEEE Journal on Selected Areas in Communications, vol. 15, no. 4, pp. 640-646, 1997.

[16] E. Panayırcı and H. A. Çırpan, "Channel estimation for spacetime block coded OFDM systems in the presence of multipath fading," in Proceedings of IEEE Global Telecommunications Conference (GLOBECOM '02), vol. 2, pp. 1157-1161, Taipei, Taiwan, November 2002, A17.

[17] S. M. Kay, Fundamentals of Statistical Signal Processing: Estimation Theory, Prentice Hall, Englewood Cliffs, NJ, USA, 1993.

[18] M. Morelli and U. Mengali, "A comparison of pilot-aided channel estimation methods for OFDM systems," IEEE Transactions on Signal Processing, vol. 49, no. 12, pp. 3065-3073, 2001.
[19] H. L. Van Trees, Detection, Estimation and Modulation Theory, Part I, Wiley Interscience, New York, NY, USA, 1993.

[20] O. Edfors, M. Sandell, J.-J. Van de Beek, S. K. Wilson, and P. O. Borjesson, "OFDM channel estimation by singular value decomposition," IEEE Transactions on Communications, vol. 46, no. 7, pp. 931-939, 1998.

[21] H. Şenol, H. A. Çırpan, and E. Panayırcı, "A low-complexity KL-expansion based channel estimator for OFDM systems," EURASIP Journal on Wireless Communications and Networking, vol. 2005, no. 2, pp. 163-174, 2005.

[22] Y. (G.) Li, L. J. Cimini Jr., and N. R. Sollenberger, "Robust channel estimation for OFDM systems with rapid dispersive fading channels," IEEE Transactions on Communications, vol. 46, no. 7, pp. 902-915, 1998.

Habib Şenol received the B.S. and M.S. degrees from the University of Istanbul in 1993 and in 1999, respectively. He is currently a Ph.D. Student at the Department of Electronics Engineering at Ișk University. From 1996 to 1999, he was a Research Assistant with the University of Istanbul. In 1999, as a Lecturer, he joined the faculty of the Department of Computer Engineering at Kadir Has University. His general re-

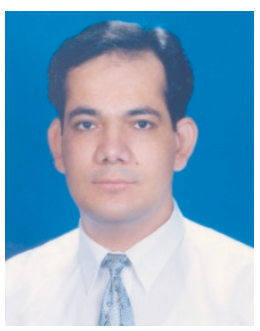
search interests cover communication theory, estimation theory, statistical signal processing, and information theory. His current research activities are focused on wireless communication concepts with specific attention to channel estimation algorithms for multicarrier(OFDM) systems. He is a Student Member of IEEE.

Hakan Ali Çurpan received the B.S. degree in 1989 from Uludag University, Bursa, Turkey, the M.S. degree in 1992 from the University of Istanbul, Istanbul, Turkey, and the Ph.D. degree in 1997 from the Stevens Institute of Technology, Hoboken, NJ, USA, all in electrical engineering. From 1995 to 1997, he was a Research Assistant with the Stevens Institute of Technology, working on signal processing algorithms for wireless

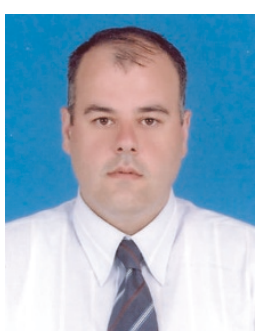
communication systems. In 1997, he joined the faculty of the Department of Electrical-Electronics Engineering at the University of Istanbul. His general research interests cover wireless communications, statistical signal and array processing, system identification and estimation theory. His current research activities are focused on signal processing and communication concepts with specific attention to channel estimation and equalization algorithms for space-time coding and multicarrier(OFDM) systems. He received the Peskin Award from Stevens Institute of Technology as well as the Professor Nazim Terzioglu Award from the Research Fund of the University of Istanbul. He is a Member of IEEE and Member of Sigma Xi.

Erdal Panayırcı received the Diploma Engineering degree in electrical engineering from the Istanbul Technical University, Istanbul, Turkey, in 1964 and the Ph.D. degree in electrical engineering and system science from Michigan State University, East Lansing, in 1970. From 1970 to 2000, he was with the faculty of Electrical and Electronics Engineering Department, Istanbul Technical

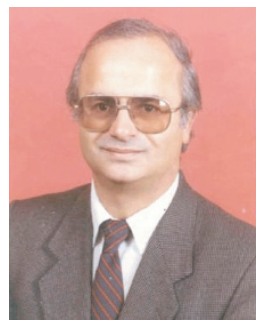


University, where he was a Professor and the Head of the Telecommunications Chair. He has also been a Part-Time Consultant to several leading companies in telecommunications in Turkey. From 1979 to 1981, he was with the Department of Computer Science, Michigan State University, as a Fulbright-Hays Fellow and a NATO Senior Scientist. Between 1983 and 1986, he served as a NATO Advisory Committee Member for the Special Panel on Sensory Systems for Robotic Control. From August 1990 to December 1991, he was a Visiting Professor at the Center for Communications and Signal Processing, New Jersey Institute of Technology, Newark, and took part in the research project on interference cancellation by array processing. Between 1998 and 2000, he was a Visiting Professor at the Department of Electrical Engineering, Texas A\&M University, College Station, and took part in research on developing efficient synchronization algorithms for orthogonal frequency-division multiplexing (OFDM) systems. He is currently a Visiting Professor at the Department of Electrical and Electronics Engineering, Bilkent University, Ankara, Turkey. He is engaged in research and teaching in digital communications and wireless systems, equalization and channel estimation in multicarrier (OFDM) communication systems, and efficient modulation and coding techniques (TCM and turbo coding). Professor Panayırc1 is a Member of Sigma Xi. He was the Editor for the IEEE Transactions on Communications in the fields of synchronization and equalizations from 1995 to 1999 . He is currently the Head of the Turkish Scientific Commission on Signals, Systems, and Communications of the International Union of Radio Science.

Mesut Çevik received the B.S. and M.S. degrees in electronics engineering from the University of Istanbul, Istanbul, Turkey, 1994 and 1996, respectively. He also received the M.S. degree in electrical engineering from Columbia University, New York City, NY, USA, in 2001. He is currently pursuing the Ph.D. degree from the Department of Electrical-Electronics Engineering, University of Istanbul, Istanbul,Turkey. His

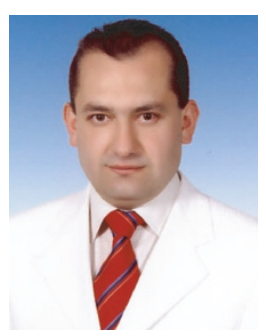
research interests lie in the areas of signal processing and wireless communications, including multicarrier(OFDM) modulation, space-time codes, and MIMO systems. 